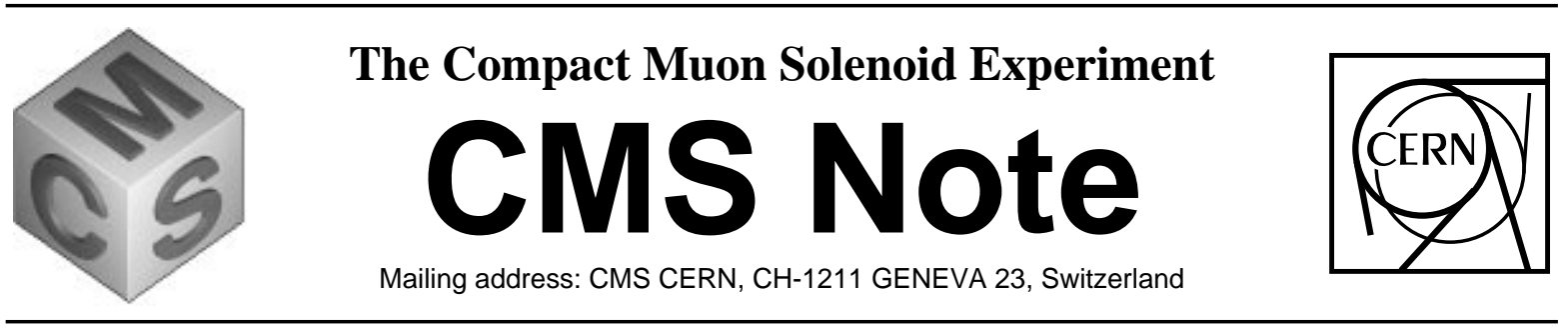

\title{
Beam Test Results for Single- and Double-Sided Silicon Detector Prototypes of the CMS Central Detector
}

\author{
O. Adriani ${ }^{5)}$, E. Babucci ${ }^{8)}$, N. Bacchetta ${ }^{7)}$, G. Bagliesi ${ }^{9)}$, P. Bartalini ${ }^{8)}$, A. Basti $^{9)}$, G. M. Bilei ${ }^{8)}$, D. Boemi ${ }^{4}$, \\ L. Borrello ${ }^{4)}$, F. Bosi ${ }^{9)}$, R. Castaldi ${ }^{9)}$, G. Castellini ${ }^{6)}$, E. Catacchini ${ }^{5)}$, U. Cazzola ${ }^{9)}$, B. Checcucci ${ }^{8)}$, \\ C. Civinini ${ }^{5)}$, D. Creanza $^{3)}$, R. D'Alessandro ${ }^{5)}$, M. de Palma ${ }^{3)}$, R. Dell'Orso ${ }^{9)}$, R. Della Marina ${ }^{2)}$, L. Fiore ${ }^{3)}$, \\ E. Focardi ${ }^{5)}$, A. Gabbanini ${ }^{6)}$, A. Giassi ${ }^{9}$, A. Giraldo ${ }^{7)}$, L. Gorn ${ }^{11)}$ M. Loreti ${ }^{7)}$, G. Maggi $i^{3)}$, G. Mantovani ${ }^{8}$, \\ M. Meschini ${ }^{5)}$, A. Messineo ${ }^{9)}$, S. My ${ }^{3)}$, G. Parrini ${ }^{5)}$, M. Pieri ${ }^{5)}$, F. Raffaelli ${ }^{9)}$, G. Raso ${ }^{3)}$, A. Santocchia ${ }^{8)}$, \\ E. Scarlini ${ }^{5)}$, G. Selvaggi ${ }^{3)}$, L. Servoli ${ }^{8)}$, L. Silvestriis ${ }^{3)}$, A. Skassyrskaia ${ }^{9}$ a), F. Szoncso ${ }^{1)}$, P. Tempesta ${ }^{3)}$, \\ M. Tesi ${ }^{6)}$, G. Tonelli ${ }^{9,10)}$, C. Vannini ${ }^{9)}$, P. G. Verdini ${ }^{9)}$, R. Wheadon ${ }^{9)}$,
}

\begin{abstract}
We report the results of two beam tests performed in July and September 1995 at CERN using silicon microstrip detectors of various types: single sided, double sided with small angle stereo strips, double sided with orthogonal strips, double sided with pads. For the read-out electronics use was made of Preshape32, Premux128 and VA1 chips. The signal to noise ratio and the resolution of the detectors was studied for different incident angles of the incoming particles and for different values of the detector bias voltage. The goal of these tests was to check and improve the performances of the prototypes for the CMS Central Detector.
\end{abstract}

to be submitted to Nucl. Instr. Meth.

\footnotetext{
1) CERN Geneva, Switzerland

2) ETH Zurich, Switzerland

3) I.N.F.N. and University of Bari, Italy

4) I.N.F.N. and University of Catania, Italy

5) I.N.F.N. and University of Florence, Italy

6) I.N.F.N. and Istituto Ricerca Onde Elettromagnetiche of Florence, Italy

7) I.N.F.N. and University of Padova, Italy

8) I.N.F.N. and University of Perugia, Italy

9) I.N.F.N. and University of Pisa, Italy

10) University of Sassari, Italy

11) Iowa State University, USA

a) On leave of absence from BINP, Moskow, Russia
} 


\section{Contents}

1 Introduction 2

2 Detectors 2

3 Read-out Electronics $\quad 3$

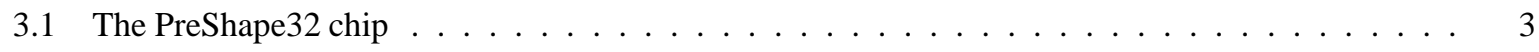

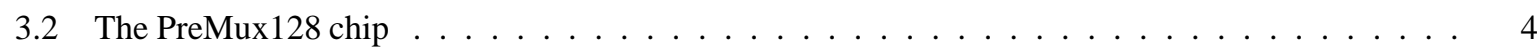

4 Detector Modules $\quad 4$

5 Experimental Set-up $\quad 5$

5.1 July' 95 beam test . . . . . . . . . . . . . . . . . . . . . . . 5

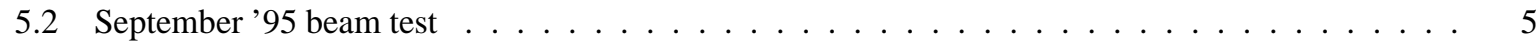

6 Data Acquisition System 5

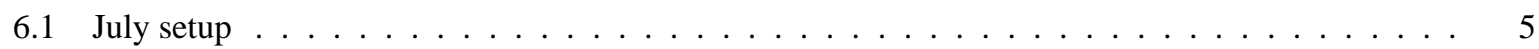

6.2 September setup . . . . . . . . . . . . . . . . . . . . . 6

7 Offline Analysis $\quad 6$

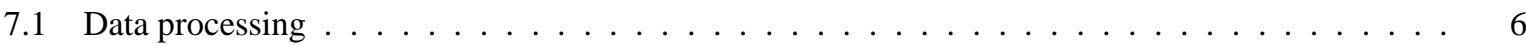

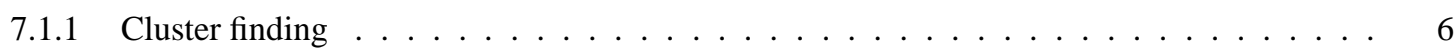

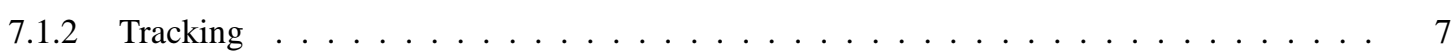

7.2 Monte Carlo . . . . . . . . . . . . . . . . . . . . . . . . . . 7

8 Results $\quad 8$

8.1 Single-sided detectors and p-side performance in general . . . . . . . . . . . . . . . . 8

8.1.1 Charge response and signal-to-noise ratio . . . . . . . . . . . . . . 8

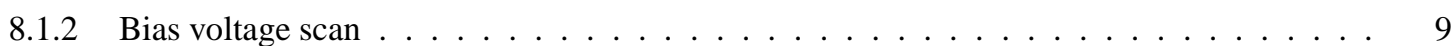

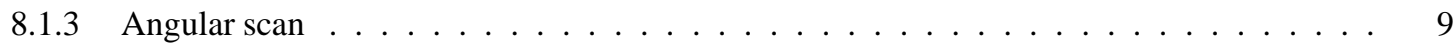

8.1.4 Response function and space resolution . . . . . . . . . . . . . . . . . . . 9

8.2 Double-sided stereo detectors and n-side performance in general . . . . . . . . . . . . . . 10

8.2.1 Charge response and signal-to-noise ratio . . . . . . . . . . . . . . . . 10

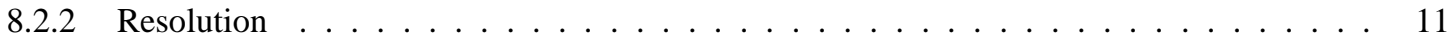

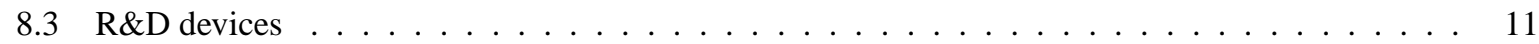

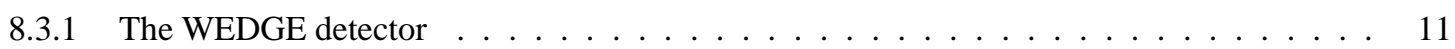

8.3.2 The DSODM and the BABY detectors . . . . . . . . . . . . . . . . 12

8.3.3 The PAD detector . . . . . . . . . . . . . . . . . . . . 12

8.3.4 Charge response in presence of weakly coupled floating strips . . . . . . . . . . . 13

9 Summary and Conclusions $\quad 14$ 


\section{Introduction}

The proposed CMS Silicon Tracker consists of four layers of silicon detectors instrumenting the intermediate part of the tracking cavity, between the inner pixel detector and the outer MSGC layers [1]. High precision microstrip silicon detectors will provide accurate measurements in the bending plane. In the barrel region, rectangular detectors with p-strips oriented along the beam direction are expected to provide point resolution better than $15 \mu \mathrm{m}$ in the $\mathrm{r}-\phi$ plane for perpendicular incidence; a coarse measurement $(\sigma \simeq 1 \mathrm{~mm})$ of the $\mathrm{z}$ coordinate provided by doublesided detectors with stereo strips on the n-side is also foreseen for pattern recognition and to resolve different interaction vertices at full luminosity. Similarly, in the forward region wedge-shaped detectors will provide precision measurements of the azimuthal coordinate and a coarse measurement of the radial coordinate. Four basic types of silicon detectors are therefore included in the CMS baseline tracker layout: single- and double-sided devices, in two different geometries (barrel-rectangular and forward-wedge).

The purpose of the beam test activity described herein was to check carefully the performances of non-irradiated single- and double-sided devices in terms of signal-to-noise ratio and space resolution under different conditions of bias voltage and for tracks at various incident angles.

Fast amplifiers (PreShape32 and PreMux128) based on the same preamplifier-shaper design that will be part of the final chip foreseen for LHC were used to study the performance of the system. Also, for a better understanding of the detector response a few detectors were read-out with conventional slow electronics (VA1).

The aim was to show that the performances attainable with non-irradiated devices coupled to the first prototypes of the final electronics are compatible with the design parameters contained in the CMS Technical Proposal. In addition, several interesting new devices were tested within an R\&D effort aimed at an optimisation of the performance of the tracker in its entirety.

\section{Detectors}

Several different types of detectors were tested on the beam. All detectors were built from standard n-type high resistivity silicon wafers, $300 \mu m$ thick. All single- and double-sided microstrip detectors tested have identical structures on the p-side. Each strip is AC coupled to external amplifiers by means of integrated capacitors built on the wafer itself by deposition of thin dielectric layers. Bias is provided to the strips via polysilicon resistors connected to the guard-ring structure. A similar arrangement is used on the ohmic side of the double-sided devices with the addition of an isolation p-stop box surrounding each electrode. In the devices featuring what we will call in the following "Double-Metal technology" (DM) the ohmic electrodes are connected, through a small contact, with a set of metal electrodes deposited on top of a thick insulator layer. The detectors tested were produced in two different technologies $[2,3,4]$ by the CSEM and SINTEF foundries. A detailed description of the technological aspects of the detectors can be found in ref. [5].

The characteristics of the detectors used are summarised in table 1, where the values following the $p$ or $n$ symbols indicate quantities pertinent to the junction or ohmic side respectively; SS stands for Single-Sided, DS for DoubleSided and DM for Double-Metal. $\mathbf{N}$ is the number of strips on the junction or ohmic side, $\mathbf{L}$ their length, $\mathbf{W}$ their width, $\mathbf{P}_{\mathbf{p h}}$ the pitch, $\mathbf{R B}$ the bias resistor value range, and $\mathbf{D}_{\mathbf{p h}}$ indicates the overall size of the detector. Note that for some double-metal detectors the pitch of the readout strips may not coincide with the pitch $\mathbf{P}_{\mathbf{p h}}$ of the charge-collection strips once the detectors are assembled to form a module, due to the possible presence of floating strips.

CSEM single-sided detectors have a large implant and metal pad on the ohmic side to provide uniform biasing, surrounded by a guard ring structure. The DS Orthogonal DM detector has 384 strips patterned on the ohmic side in the Metal-2 layer on a decoupling dielectric consisting of $4 \mu m$ thick Silicon dioxide. These readout strips are orthogonal to the detector strips and connected to them by vias; the BABY detector is another double-sided, orthogonal, double-metal design, and it has a Metal-2 pattern on the ohmic side consisting of 186 strips orthogonal to the $n^{+}$implants. The so-called stereo detectors are double-sided detectors where the strips on the ohmic side are tilted with an angle of $100 \mathrm{mrad}$ with respect to those on the junction side. Fig. 1 shows the layout of the ohmic side strips for the SINTEF Double-Sided Stereo Double-Metal detectors. These detectors have an additional Metal-2 pattern of $25.7 \mathrm{~mm}$ long strips on the ohmic side that run orthogonal to the junction side strips; each of them is connected to two detector strips at the edges of the rectangular active area, by vertical vias passing through a decoupling dielectric consisting of a $4 \mu \mathrm{m}$ thick polyimide layer. This arrangement was used to avoid dead zones in the two triangular areas at the opposite edge of the module. The PAD detector has the ohmic side patterned into 4 columns of 32 pads, each $6.4 \times 2 \mathrm{~mm}^{2}$. For the WEDGE detector (fig. 2) the strips of the p-side follow the 
Table 1: Detector Characteristics

\begin{tabular}{|l|l|l|cccccc|}
\hline Detector & Maker & & $\mathbf{N}$ & $\mathbf{L}$ & $\mathbf{W}$ & $\mathbf{P}_{\mathbf{p h}}$ & $\mathbf{R B}$ & $\mathbf{D}_{\mathbf{p h}}$ \\
$(\mathrm{mm})$ & $(\mu m)$ & $(\mu m)$ & $(\mathrm{M} \Omega)$ & $\left(\mathrm{mm}^{2}\right)$ \\
\hline SS Small Size & CSEM & $p$ & 384 & 62.2 & 14 & 50 & $0.7-1.4$ & $21 \times 64$ \\
\hline SS Full Size & CSEM & $p$ & 1024 & 62.2 & 14 & 50 & $0.7-1.4$ & $64 \times 64$ \\
\hline DS Stereo & CSEM & $p$ & 384 & 62.2 & 14 & 50 & $0.7-1.4$ & $21 \times 64$ \\
& & $n$ & 256 & 62.2 & 28 & 100 & $0.7-1.4$ & \\
\hline DS Orthog DM & CSEM & $p$ & 384 & 62.2 & 14 & 50 & $0.7-1.4$ & $21 \times 64$ \\
& & $n$ & 621 & 19.2 & 28 & 100 & $0.7-1.4$ & \\
\hline BABY & CSEM & $p$ & 384 & 8.2 & 14 & 50 & $\sim 1.0$ & $21 \times 10$ \\
& & $n$ & 186 & 19.2 & 14 & 50 & $\sim 0.7$ & \\
\hline DS Stereo DM & SINTEF & $p$ & 512 & 62.5 & 14 & 50 & $0.3-1.0$ & $30 \times 65$ \\
& & $n$ & 256 & $\leq 65$. & 28 & 100 & $0.3-1.0$ & \\
\hline PAD & SINTEF & $p$ & 512 & 62.5 & 14 & 50 & $0.3-1.0$ & $30 \times 65$ \\
& & $n$ & 32 & 6.4 & 2000 & & $0.3-1.0$ & \\
\hline WEDGE & CSEM & $p$ & 128 & 62 & $14-26$ & $38-50$ & 18 & $8.6 \times 64$ \\
& & $n$ & 495 & $4.8-6.3$ & 60 & 125 & 25 & \\
\hline
\end{tabular}

trapezoidal geometry with a fanning structure that makes the pitch change from 38 to $50 \mu \mathrm{m}$. The $\mathrm{n}$-side strips have a pitch of $125 \mu \mathrm{m}$ and are orthogonal to the central strip of the p-side.

In the CSEM process a typical yield for coupling capacitors was found to be $\sim 1 \%$ shorted capacitors on the junction side and $\sim 3.5 \%$ on the ohmic side; the typical leakage current density for these devices were below $50 \mathrm{nA} \mathrm{cm}{ }^{-2}$ at $100 \mathrm{~V}$. The SINTEF devices gave a yield of $\sim 1 \%$ broken capacitors on the junction side, $\sim 2 \%$ for the ohmic side and typical leakage current density at $100 \mathrm{~V}$ of $\sim 350 \mathrm{nA} \mathrm{cm}^{-2}$.

\section{Read-out Electronics}

For the two test periods in which the data for this study were taken, two different readout chips, and consequently two different readout systems, were tested. Both chips were developed by the RD20 Collaboration $[4,6,7,8]$ and are based on the same preamplifier-shaper design used in the APV-series chips [9], meant for LHC tracker applications. Initially only the PreShape 32 chip [10] was available and was therefore used. Later, when the multiplexed PreMux128 chip [11] became available it replaced the PreShape32.

In order to decouple from the tests possible effects of the relatively new RD-20 chips, a small number of detectors were also tested with LEP-type electronics, namely the VA1 chips. These are characterized by a peaking time of about 1 to $2 \mu$ s and a very low Equivalent Noise Charge figure of $\sim 140 e^{-}+12 e^{-} / \mathrm{pF}$ (of course with LHC-type detectors the noise from the relatively low value of the bias resistors and eventually from the high dark currents after irradiation would dominate). The VA1 chips were also used for the B1, B2 and B3 detectors in the beam telescope [12].

\subsection{The PreShape32 chip}

The PreShape32 contains 32 channels of integrated preamplifier/shaper circuits optimised for MSGC detector signals, even though they are quite usable with silicon detectors as well. Each channel consists of a charge preamplifier followed by a shaping amplifier which can be adjusted, via externally provided bias currents and voltages, to provide a simple CR-RC type pulse with a nominal peaking time of $45 \mathrm{~ns}$. The 32 inputs at a $100 \mu m$ pitch are DC-coupled to the preamplifiers, since the design was intended to be used with silicon detectors incorporating capacitive coupling on the detector wafer. The output of each shaper is followed by a single-ended buffer, which drives the signal off chip. For nominal bias currents, linearity is reasonable over the range $\pm 25 \mathrm{fC}$, or \pm 6 times the charge deposited by a minimum ionising particle in $300 \mu \mathrm{m}$ of Silicon. In order to match the $220 \Omega$ impedance of the PreShape 32 outputs to the $50 \Omega$ cables, and also to better drive the $30 \mathrm{~m}$ long cables going to the counting room, special single-ended buffer amplifiers [13] were developed by IPN Lyon. 


\subsection{The PreMux128 chip}

Readout of a large number of strips of detectors connected to PreShape 32 chips, requiring a separate ADC channel for each strip, proved to be a major problem. However, the PreMux128 expansion of the PreShape32 solved this problem by adding multiplexing of the analogue outputs. A PreMux128 chip contains 128 channels of charge preamplifiers and shaper-amplifiers analogous to the PreShape32 design, plus 128 channels of double correlated sampling circuitry and an analogue multiplexer. This made the use of Flash-ADC boards possible, with a single FADC channel reading out an entire detector module, and with some additional circuitry to handle the delicate aspects of token-passing and output buffering, even multiple modules could be read with a single FADC channel, at the expense of course of a longer digitization time per event.

The PreMux128 multiplexer allows two possible modes of operation in order to subtract baseline fluctuations directly on-chip: a "double-correlated sampling" mode in which for each channel the output is the difference between the value sampled at trigger time and a "pre-trigger" sample, and a single-sampling mode, better suited for asynchronous operation, in which the baseline value to be subtracted comes from an additional preamplifier and shaper, isolated from the inputs. The use of the double-correlated sampling mode having the added disadvantage of an increased noise (by a factor $\sqrt{2}$ ), all data were collected by operating the PreMux chips in the alternative single-sampling mode.

\section{Detector Modules}

Detectors and electronics units have been assembled together in different detector modules as indicated in table 2, where $\mathbf{L}$ indicates the total strip length in the module and $\mathbf{P}$ the read-out pitch. When two detectors were used to built a module, the procedure consisted in glueing them together under a coordinate measuring machine with a relative alignment between the two detectors better than $3 \mu \mathrm{m}$ in the directions parallel and orthogonal to the strips and a planarity better than $20 \mu \mathrm{m}$.

Table 2: Modules

\begin{tabular}{|l|l|l|c|c|c|l|}
\hline Module Name & Detectors & & $\begin{array}{c}\mathbf{L} \\
(\mathrm{mm})\end{array}$ & $\begin{array}{c}\mathbf{P} \\
(\mu \mathrm{m})\end{array}$ & $\begin{array}{c}\text { Floating } \\
\text { Strip }\end{array}$ & Electronics \\
\hline \hline SS & 1 SS Small Size & $p$ & 62.2 & 50 & & VA1 \\
\hline SSFS & 2 SS Full Size & $p$ & 124.4 & 50 & & Premux128 \\
\hline DSS & 1 SS Small Size and & $p$ & 124.4 & 50 & & Preshape32 \\
& 1 DS Stereo & $n$ & 62.2 & 100 & & Preshape32 \\
\hline DSODM & 1 DS Orthog DM & $p$ & 62.2 & 50 & & Premux128 \\
& & $n$ & 19.2 & 100 & & Premux128 \\
\hline BABY & 1 BABY & $p$ & 8.2 & 100 & Yes & VA1 \\
& & $n$ & 19.8 & 50 & & VA1 \\
\hline DSSDM & & $p$ & 125. & 50 & & Premux128 \\
& 2 DS Stereo DM & $n$ & 138. & 100 & & Premux128 \\
\hline DSSDM & & $p$ & 125. & 50 & & Premux128 \\
& 2 DS Stereo DM & $p$ & 138. & 200 & Yes & Premux128 \\
\hline PAD & 2 PAD & $n$ & & & & Premux128 \\
\hline WEDGE & 1 WEDGE & $p$ & 62 & $38-50$ & & Premux128 \\
& & $n$ & $4.8-6.3$ & 125 or 250 & Mixed & Premux128 \\
\hline
\end{tabular}

The strips coupled to the small fraction of broken capacitors were not connected to the read-out electronics.

The depletion voltage was always applied through the ohmic side; in case of double-sided modules the ohmic side electronics was therefore operated around a virtual ground corresponding to the bias voltage. 


\section{Experimental Set-up}

\subsection{July '95 beam test}

The set-up of the beam test performed in July '95 in the H2 beam line of the CERN SPS North Area is shown in fig. 3 .

A description of the beam test area and of the trigger system used to select a $300 \mathrm{GeV} / \mathrm{c}$ muon beam can be found in [14]. The magnetic field was off throughout the tests described in this paper.

Several detectors of known performances, grouped as $B_{1}, B_{2}$ and $H_{1}$, were used as reference telescopes to precisely reconstruct the trajectories of incoming particles.

$B_{1}$ and $B_{2}$ each consisted of two identical double-sided silicon microstrip detectors, with orthogonal strips, of area $1.92 \times 1.92 \mathrm{~cm}^{2}$ and thickness $300 \mu \mathrm{m}$ [12]. Each of them had 384 read-out strips with $50 \mu \mathrm{m}$ pitch on each side, and a floating strip between two read-out strips on the junction side. The spatial resolution was $3 \mu m$ on the junction side and $6 \mu \mathrm{m}$ on the ohmic side. Read-out was performed using VA1 chips and VME SIROCCO-1 modules.

$H_{1}$ was made of eight single-sided silicon detectors, with 512 strips $5.82 \mathrm{~cm}$ long and a thickness of $500 \mu \mathrm{m}$ [15]. The read-out pitch was $50 \mu \mathrm{m}$ and one floating strip for each read-out strip was present. The planes were arranged along the beam line in such a way that the detectors alternately measured the vertical or the horizontal coordinate of the incoming particles. Their spatial resolution was $6 \mu \mathrm{m}$. Read-out was performed using MX-2 chips and dedicated digitizing and clustering VME boards.

Between these telescopes one optical bench of $1 \mathrm{~m}$ length supported several modules equipped with MSGC, MGC, Silicon and GaAs detectors, mounted on frames that allowed for rotation around an horizontal axis perpendicular to the beam. Here we will discuss results relative to the following silicon detectors: a Double Sided Stereo (DSS), a Single Sided (SS) and a Double Sided Orthogonal Double Metal (BABY).

The results exposed hereafter are based on a sample of about $400 \mathrm{~K}$ triggers taken during this beam test.

For some detectors angular and bias voltage scan were performed. In those cases a minimum of $8 \mathrm{~K}$ events per point was recorded at incident angles ranging from $5^{\circ}$ to $20^{\circ}$ and at different bias voltage settings.

\subsection{September'95 beam test}

In September' 95 , the test was performed on the X7 beam line at the CERN SPS West Area, using a pion beam with energies of 20 and $50 \mathrm{GeV} / c^{2}$. For this test one more telescope, $B_{3}$, was added: it consisted of two double-sided detectors having the same characteristics as those of $B_{1}$ and $B_{2}$, except the bigger active area $\left(5.26 \times 5.26 \mathrm{~cm}^{2}\right.$ with 1024 read-out strips on each side).

The detectors under test were arranged along the beam line on two optical benches between the telescopes, as shown in fig. 4.

Here we will report results for the following detectors: a Double-Sided Pad (PAD), a Single-Sided Full Size (SSF), two Double-Sided Stereo (DSSDM 100 and DSSDM 200 ), a Double-Sided Orthogonal Double-Metal (DSODM) and a Double-Sided Wedge (WEDGE).

In september, for all detectors the read-out was performed using PreMux 128 chips.

A total of about $500 \mathrm{~K}$ triggers were taken including angle and bias voltage scans.

\section{Data Acquisition System}

\subsection{July setup}

During the July '95 beam test the data acquisition was based on the RD5 framework [16]. All modules equipped with PreShape32 electronics were read out using LeCroy 2280 CAMAC Charge ADC boards. These devices were operated in a "wide gate" mode, integrating the CR-RC shaped pulses from the PreShape32 chips (which have a nominal risetime of $45 \mathrm{~ns}$ ) over a period of approximately $500 \mathrm{~ns}$. This was necessary because the ADC characteristics did not allow for operation with a sufficiently short integration gate to sample the CR-RC pulse peak only. Moreover, the board inputs being unipolar and having an impedence of $50 \Omega$ required the use of a large number of high speed, high density buffer hybrids to drive the long BNC cables from the detectors to the counting room. Altogether, this resulted in increased noise and sometimes in high frequency oscillations with an overall degradation of the detector performances with respect to the a priori expectations. 
The CAMAC crates housing the LeCroy ADCs were also equipped with a LeCroy Scanning Processor, which did some zero-suppression before the data was transferred to a VME-based MC68020 CPU which handled the event protocol and the further data transfer to the RD5 Event Builder.

Those detectors that were equipped with VA1 chips, as well as the B1 and B2 telescopes, having a serialized output could be read out using a LEPSI-designed VME Flash ADC/Transient Digitizer, the SIROCCO-1 [17] capable of sampling up to 4096 "buckets" at a conversion rate of up to $5 \mathrm{MHz}$, with 10-bit accuracy and a programmable baseline adjustment for optimal use of the $\pm 300 \mathrm{mV}$ input range.

The FADC boards were controlled and read out by MC68020 cards, and the data transferred over VSB/VME Dual Port Memories (DPM) to the Event Builder crate for logging and monitoring.

The digitization and cluster finding for the $H_{1}$ telescope was done by ROXI, dedicated VME Flash ADC cards with DSP56001 processors on board. Cluster information was then transferred to a dedicated processor card with a MC68040 CPU over a number of TAXI links. Once more, data were transferred to the RD5 Event Builder via a Dual Port Memory. For a detailed description of the digitizer/clustering boards and of the event builder see ref. [15].

\subsection{September setup}

For the X7 test in September '95 a completely new system was assembled from scratch in a big effort to provide DAQ capabilities completely independent from the RD5 system. No CAMAC digitizers were used, and all detectors, which were by then equipped with PreMux 128 electronics, were read out by either SIROCCO-1 or the functionally analogous CAEN V551 FADC boards.

The WEDGE and DSODM read out system [18] used different ADCs and an extensive decoupling schemes allowing a fully floating connection to the detectors. The analog signal from the PreMux 128 was converted by flash ADC boards placed next to the detectors. The data coming from the ADC were immediately moved in FIFO memories, then serially transferred to a VME crate and written into a DPM which interfaced the system to the main DAQ. The WEDGE and DSODM VME crate consisted basically of two Motorola CPU boards, one having a full Unix operating system which handled disks and networking, while the other was dedicated to the running of binaries compiled and downloaded by the control CPU. The sequences needed to drive the PreMux128, the ADC strobe and the control logic for the FIFO's, and the serial communication have been implemented using the Altera family of programmable devices. This part of DAQ could be separately controlled and monitored using a HP workstation, with the added possibility of an almost real time data analysis.

The multi-crate system used MC68040 processors running the OS-9 operating system for the front-end crates, where the digitizers and the sequencers controlling the electronics and the ADCs are located, and a VME-based Sparc 5/64 processor in the Event Builder crate. A simple handshake protocol handling dual "ping-pong" buffers, implemented using DPM boards, dealt with the data transfer from the front-end crates to the Event Builder. Data from the detectors were digitized and buffered in the MC68040 "private" memory throughout the SPS spill. An end-of-spill interrupt to the processors triggered the data transfer to the DPMs, finally signaling the availability of the data to the Event Builder CPU. This in turn handled the logging of Zebra banks to tape and some online monitoring of the detector performance.

\section{Offline Analysis}

\subsection{Data processing}

\subsubsection{Cluster finding}

The signal $S^{(i)}$ on the $i$-th strip at each event is extracted from the value read from the analog-to-digital converter, $A D C^{(i)}$, by subtracting the strip pedestal $P E D^{(i)}$ and the common mode fluctuation for the event, $C M$ :

$$
S^{(i)}=A D C^{(i)}-P E D^{(i)}-C M
$$

The common mode represents the joint shift of the baseline in a group of strips situated in the same readout unit, possibly caused by low frequency pickup. The $C M$ is defined for each event and is computed for sets of contiguous strips situated on the same physical chip using an iterative trimmed mean algorithm.

The pedestal of each strip is defined as the average of the $A D C$ counts of that channel, evaluated and updated every 100 events, taking care not to include those events in which the strip may have been hit by a particle. On the same sample of 100 events, the strip noise is also calculated as the statistical fluctuation of the signal $S^{(i)}$. 
Strips are identified as bad (noisy, disconnected or shorted) when the value of their noise goes outside an acceptance range; as soon as they are flagged they are excluded from further analysis. The first 50 events are used only to calculate the starting values for the pedestal and noise of each channel.

To cross-check the results of this method we have developed a different algorithm that uses an iterative procedure to find the pedestals, the noise and the bad strip list. The pedestal value is still defined as the average of the $A D C(i)$ counts but the events over which it is computed are now all the events of the run in which the $i$-th strip has not been hit by a particle (class $C^{(i)}$ ). This class is selected for each iteration checking if the $S^{(i)}$ differs from zero by at least three times the value of the noise for the particular channel. The noise $\left(N^{(i)}\right)$ is defined as the root mean square of $S^{(i)}$ in the class $C^{(i)}$, except for the first iteration where it is set to zero. Is it clear that at the end of the first iteration $P E D^{(i)}$ and $N^{(i)}$ have only approximative values since at that stage all the events in the run are used in $C^{(i)}$. After few iterations the procedure converges and a list of bad strips is obtained for all the channels whose $N^{(i)}$ is outside a certain range. Both methods give comparable results.

Using the previous definitions for the signal and the noise of each strip we then look for clusters of adjacent strips in order to identify the passage of a particle.

The offline search algorithm looks for contiguous strips having a signal satisfying $S^{(i)} / N^{(i)}>T_{0}$, with $T_{0}=3$ or more depending on the specific detector examined. Once at least one such strip is found, all adjacent strips with $S^{(j)} / N^{(j)}>T_{1}$ are added to the cluster, with a minimum value of $T_{1}=2$. When no more adjacent strips above threshold are found, the cluster charge $S^{\text {cluster }}$ is computed as the sum of the pulse heights over the accepted strips. The cluster noise is then defined as $N^{\text {cluster }}=\sqrt{\sum_{\text {cluster }}\left[N^{(i)}\right]^{2} / L^{\text {cluster }}}$, where $L^{\text {cluster }}$ is the number of strips accepted for that cluster. If the condition $S^{\text {cluster }} / N^{\text {cluster }}>T_{2}$ is satisfied, the cluster is retained. Typical values for $T_{2}$ for the various detectors range from 5 to 10 . The performance of the detectors was found to be reasonably stable for $10 \%$ variations of the threshold values.

The position is then calculated from the accepted clusters as the pulse height-weighted mean of all strips.

\subsubsection{Tracking}

To determine the spatial resolution of the detectors under test use was made of the tracking information provided by the beam telescopes. For this purpose, all the system must be properly aligned first.

The alignment procedure computes, for each detector, the three translational offsets in $x, y$ and $z$ and the rotation angle $\phi$ in the plane orthogonal to the nominal beam direction $(z)$; the other possible rotations were found to give negligible effects.

For each event the straight line $L$ joining the hits in the first and in the last telescope detector is found. The other telescope planes and the test detectors are aligned looking at the distribution of the residuals between the crossing point of $L$ with each detector plane and the locally reconstructed hit position. The values of the rotation angle $\phi$ and of the $z$ offset are found minimising the width of the residual distribution for each module, while the $x$ and $y$ offsets are given by the average of the distribution itself.

Completely consistent results were obtained using a different alignment procedure: the points measured by all detectors are used to reconstruct two straight lines (least square fit), one for the $x$ and one for the $y$ projection. These tracks are then extrapolated to determine the impact point on the detector planes. The offsets and the rotation parameters are then determined by a global minimisation of the width of the residual distribution.

From the Monte Carlo simulation the extrapolation error on the impact point due to multiple scattering and to finite telescope resolution was found to range from $5 \mu \mathrm{m}$ to $9 \mu \mathrm{m}$ both in the $x$ and $y$ projection depending on the position along the set-up. The resolution of each detector can then be obtained by subtracting in quadrature the extrapolated impact point error from the width of the distribution of the residuals. Possible deterioration effects on the measured resolution due to remaining uncertainties in the alignment parameters are presently neglected.

\subsection{Monte Carlo}

For the Monte Carlo simulation of the test setup, the relevant routines describing the functioning of silicon detectors were extracted from the general CMSIM simulation [19] and included in a simplified GEANT skeleton. A complete geometrical description of the experimental setup and of the detectors under study was done.

The strip signal is generated as follows:

- The entry point (A), the exit point (B), and the energy deposition in the silicon $\left(E_{i o n}\right)$ by the incident particle 
are given directly by the GEANT package during the tracking simulation.

- Inside the silicon wafer, the track segment $\mathrm{AB}$ is subdivided in $N_{\text {seg }}$ subsegments (currently 40 ) and a fraction of the deposited energy $E_{\text {ion }} / N_{\text {seg }}$ is assigned to each. For each subsegment a packet of hole-electron pairs is created, the diffusion time and the related diffusion width are calculated and the deviation of the drift direction due to the eventual magnetic field is taken into account.

- For each drifted hole (electron) packet the fraction of charge collected by every strip falling in the $3 \sigma$ range of the diffusion cloud is calculated. When floating strips are present, the resulting charge distribution is modified correspondingly.

- The noise on the read-out strips is generated [20]. This is made of two components: the parallel noise which originates from the detector components and the series noise which is generated inside the amplifier. The latter also generates some noise charge on the neighbouring strips by capacitive coupling.

The parameters describing the charge division mechanism and the parallel and series noise generation were tuned to reproduce the measured cluster width, noise level and signal/noise ratio. An example of the agreement obtained is shown in fig. 5 where real data distributions from a SSFS module are superimposed to the distributions obtained by the MC simulation of a module having the same caracteristics.

Analysing MC events with the same program used to measure the resolution of real data, good agreement was found as is shown in fig. 6 for the two detectors SSFS and DSSDM 200 . The first row (figs. 6a and b) shows the detector intrinsic resolution for the two detectors, defined as the difference between the coordinate as reconstructed from the simulated charge collected by the strips and the MC "true" particle crossing point. To estimate the tracking interpolation error, due to multiple scattering and finite beam telescope resolution, figs. $6 \mathrm{c}$ and $6 \mathrm{~d}$ show the difference between the interpolated track impact point as reconstructed by the simulated beam telescope hits, and the "true" crossing point. Lastly, in figs. 6e and $6 f$ the histograms show the distribution of the residuals (i.e. the difference between the interpolated track point and the reconstructed coordinate) for simulated events, while the superimposed points refer to real beam test data.

\section{Results}

\subsection{Single-sided detectors and p-side performance in general}

\subsubsection{Charge response and signal-to-noise ratio}

In CMS baseline design the high resolution track information is provided by the strips on the junction side. For this reason, the behaviour of single-sided detectors and of the p-side of double-sided devices was studied in detail. The detector response is characterized by the set of plots shown in fig. 7. All plots refer to the typical response of the junction side in overdepleted detectors for tracks at perpendicular incidence.

The cluster shape gives us information on how the charge is shared between the strips in the same cluster. Fig. 7a shows how the "seed" strip in multi-strip clusters collects on average more than $65 \%$ of the total charge of the cluster. Any asymmetry in the distribution of this plot may indicate problems in the charge sharing that could be attributed to non-optimized performance of detectors or electronics.

The cluster multiplicity distribution, plotted in fig. $7 \mathrm{~b}$, shows that $75 \%$ of the clusters are single-strip clusters while the two-strip clusters are about $22 \%$ of the total. We expect charge division, and therefore two-strip clusters, only for tracks hitting the central region between the two strips, with the charge spread on both due to diffusion and delta rays, while for tracks in the proximity of a strip the charge is collected by that strip only.

The charge-sharing mechanism can be studied from the plot of fig. 7c which shows the correlation between the charge collected by the "left" and the "right" strips, where left and right is defined relatively to the reconstructed hit position. As expected, in part of the events the signal is collected by either the right or the left strip, and only in a fraction of the events the charge is shared.

The signal-to-noise ratio has been measured for different configurations of detectors. Of particular interest are the results for the SSFS module, which can be considered a good approximation of the final single-sided detector module of CMS. The distribution of the signal, i.e. the total charge associated with a cluster, for this module is shown in fig. $7 \mathrm{~d}$ with a Landau fit superimposed to the data.

The cluster noise, as defined in Section 7.1.1, and the signal-to-noise ratio, are shown in figs. 7e and 7f. A summary of the $\mathrm{S} / \mathrm{N}$ performance of the p-side for different detector configurations is shown in table 3 . The figures listed 
are computed as the ratio of the most probable signal value from the Landau fit to the average noise as fitted with a Gaussian. The S/N value of 26.1 for the SSFS module is in good agreement with the expected noise performance of the electronics and the charge released in $300 \mu \mathrm{m}$ thick detectors.

\subsubsection{Bias voltage scan}

On all devices we performed a voltage scan to study the effect of different bias voltages on the signal-to-noise ratio. The voltage range scanned was from 10 to 60 Volts for the SS detector, from 30 to 100 Volts for DSODM and WEDGE and from 60 to 200 Volts for SSFS. Except for very low bias voltages, the noise should stay basically constant while the signal should increase up to its maximum value at $V_{d e p}$ when the depleted region extends to the entire thickness of the wafer. This standard behaviour was observed in all detectors. Furthermore, we measured a slight improvement of the signal-to-noise ratio even after full depletion as indication of a better charge collection efficiency due to the higher electric field inside the silicon. As one may expect, the effect is more evident when fast shaping time electronics is used. Fig. 8a refers to a module read-out with relatively slow VA1 electronics, while figs. $8 \mathrm{~b}$ and $8 \mathrm{c}$ show the results for PreMux128 equipped detectors.

The data taken with the SSFS module biased at voltages up to 200 Volts are of particular interest since high voltage operation is foreseen in CMS to survive the effect of irradiation. As can be seen in fig. 9 the SSFS module itself was able to stand voltages up to 300 Volts before reaching the breakdown point; nevertheless for safety reasons we decided to limit this first test to a maximum of 200 Volts.

Fig. 10a shows the effect of bias on the $\mathrm{S} / \mathrm{N}$ ratio for this module. Although the average noise figure remains almost constant with increasing bias, we monitored the behaviour of individual channels to look for hot strips which could affect the local efficiency. In figs. $10 \mathrm{~b}$ and $10 \mathrm{c}$ we compare the individual channel noise distribution at $V_{d e p}$ and at 200 Volts. Only a small fraction of channels (1\%) appears to be affected by the high voltage operation and exhibits an increased noise, but the values reached are however still quite manageable by the clustering algorithm.

\subsubsection{Angular scan}

All data presented so far were taken exposing the detectors to the beam at normal incidence $(\alpha=0)$. The effect of inclined tracks on the response of the p-side has been studied in detail by rotating the detector modules around the horizontal axis perpendicular to the beam direction. The mechanics allowed for rotation angles up to $25^{\circ}$.

The detectors were mounted with the p-side strips parallel to the rotation axis, therefore the amount of charge released in the silicon and the cluster width are expected to increase for geometrical reasons. Consequently the signal-to-noise ratio is expected to improve with the angle.

All detectors behave as expected: there is a clear widening of the p-side clusters, an increase in the total collected charge compatible with the expected $1 / \cos \alpha$ dependence, no visible effect on noise and consequently an increase of the signal-to-noise ratio. Examples of the behaviour of the various parameters are shown in fig. 11 for the p-side of DSODM.

\subsubsection{Response function and space resolution}

To reconstruct the particle impact point from the cluster information, the center of gravity of the cluster, weighted with the charge collected on each strip, is usually evaluated. This way of reconstructing the position assumes a linear sharing of the charge released on each strip, an assumption which is far from reality in our case.

To study the deviations from the linear approximation and possibly give a correction to the reconstructed position we have studied the response function defined as $\eta=Q_{L} /\left(Q_{L}+Q_{R}\right)$, where $Q_{L}$ and $Q_{R}$ refer to the charge collected on the left and right strip respectively. In fig. $12 \mathrm{a}$ a typical $\eta$ distribution for the $\mathrm{p}$-side of a detector is shown superimposed to the equivalent MC distribution. The reconstructed position, modulo the strip pitch, is another variable which shows the non-uniformity of the reconstruction across the region between two neighbouring strips, in fig. $12 \mathrm{~d}$ this variable is plotted as a function of the value of $\eta$ for real data.

From figs. 12b, 12c, 12e and 12f, where entries are subdivided into two multiplicity classes, it is evident that in the central region, populated by multi-strip clusters, the charge is linearly shared among the strips and hence the linear interpolation is correct, while in the proximity of the strips single-strip clusters dominate and a digital response should be assumed.

The detectors were placed within a high precision telescope system to study the position resolution. For alignment studies and for position resolution measurements, only events with one cluster per view in each telescope detector 
were used. A typical distribution of the residual, i.e. the difference between the locally reconstructed position and the interpolated impact point of the beam telescope track, is plotted in fig. 13a for a standard p-side $50 \mu \mathrm{m}$ pitch device. The residuals are computed using the centre of gravity technique previously described. By subtracting in quadrature the tracking error from the width of the residual distribution we obtain the resolution values listed in table 4. In all cases the resolution is better than $15 \mu \mathrm{m}$, the goal value foreseen for the CMS detector module. The SSFS module gives, after subtracting $9 \mu \mathrm{m}$ in quadrature for the error of the track interpolation, a point resolution of $11 \mu \mathrm{m}$. The dependence of the resolution from the signal-to-noise ratio is weak: even with a most probable S/N of 8.2, as in the case of the p-side of DSS, we can achieve a space resolution of about $14 \mu \mathrm{m}$. These values of resolution can be further improved by using the non linear $\eta$-technique which makes use of the response function distribution to interpolate properly the measured coordinate. This exercise was performed for the $\mathrm{n}$-side of the BABY detector yielding an improvement in the detector resolution from $6.2 \mu \mathrm{m}$ to $4.7 \mu \mathrm{m}$.

By using the telescope information we performed a careful study of the resolution versus interstrip position (fig. 13d); the digital response of the region close to the read-out strips is clearly separated from the intermediate region dominated by charge sharing. The same behaviour is also visible in fig. $13 \mathrm{~b}$ and $13 \mathrm{c}$, where the residuals of the single- and multi-strip clusters are plotted separately. Fig. 13e shows the resolution along the strip to check for uniformity of response over the detector length.

Data taken with tilted detectors were used to study the resolution for inclined tracks. Fig. 14a shows the resolution and fig. 14d the average number of strips per cluster versus the tilt angle for the DSS module. The resolution at first improves because of the increased two-strip cluster contribution, and subsequently deteriorates due to fluctuations in the charge released on the side strips as the clusters become wider.

\subsection{Double-sided stereo detectors and n-side performance in general}

\subsubsection{Charge response and signal-to-noise ratio}

The response of the n-side has been analysed in terms of cluster shape and cluster multiplicity using the approach previously described for the p-side. The same distributions of fig. 7 are shown in fig. 15 for the $n$-side of the $\operatorname{DSSDM}_{200}$ device. The average cluster multiplicity is larger, due to the presence of the floating strips, a further effect of which is the clustering of events in which the signal is equally shared between the left and the right strips in the scatterplot of fig. $15 \mathrm{c}$.

The performance in terms of signal-to-noise ratio for the $\mathrm{n}$-side of different detector modules is summarized in table 3. It should be noticed that in general, for detectors with comparable strip length on both sides, the $\mathrm{S} / \mathrm{N}$ values for the ohmic side are lower than the corresponding values for the junction side. The difference is mainly due to the fact that all detector modules were biased through the ohmic side and several paths of possible interference between the read-out lines and the power supply circuitry were present in the motherboard coupled to the detector modules. To check this point we inverted the high voltage circuit in one detector module to bias the silicon through the $\mathrm{p}$-side and a symmetric worsening of the noise on the junction side was observed.

Particular attention was put in the evaluation of the performance of the DSSDM modules, very similar, as far as the main features are concerned, to the baseline CMS double-sided barrel modules. We were particularly interested in verifying the effects of the double-metal technology on the stereo devices and to compare the performance of identical devices read-out at a $100 \mu \mathrm{m}$ pitch with no floating strip and at a pitch of $200 \mu \mathrm{m}$ with floating strips (DSSDM $_{100}$ and DSSDM $_{200}$ modules).

The effect of the double-metal technology was evaluated: the second metal layer introduces an additional coupling capacitance which increases the total capacitance at the input of the amplifier and may also produce some charge loss. To study this problem we subdivided the strips on the ohmic sides in two samples: "DM" strips, joined across the detector through the second metal layer, and "non-DM" strips, with no contact to the second metal layer. For the two categories we compared the performance in terms of cluster charge, noise and signal-to-noise ratio. The results for the DSSDM 100 module are shown in fig. 16. We observe in fact a worsening of the $\mathrm{S} / \mathrm{N}$ ratio at the $10 \%$ level between the two categories which can be attributed to the effect of the additional capacitance. A similar behaviour is observed also in the $\operatorname{DSSDM}_{200}$ module. We consider this slight deterioration compatible with the required performance and fully compensated by the advantage of recovering the dead areas at the edges of the standard stereo devices, about $25 \%$ of the detector area for a stereo angle of $100 \mathrm{mrad}$.

The bias voltage scan gives no signal on the ohmic side until full depletion is reached and the isolation mechanism among the n-strips becomes effective; a slight improvement of the signal-to-noise ratio above $V_{\text {dep }}$ is still visible (fig. 17), but the effect of a higher electric field is less important for electrons whose mobility is already high enough to cope with the $45 \mathrm{~ns}$ peaking time of the read-out chips. The effects of the angular scan in terms of 
collected charge are similar to those previously described for the junction side.

The charge sharing mechanism on the ohmic side was studied for all devices with a particular attention to the effects of large read-out pitches. The effect of the floating strip can be easily seen from fig. 18 where the Response Functions and their correlation with the interstrip position are compared for the two detectors DSSDM 100 and $\mathrm{DSSDM}_{200}$.

The charge response dependence on the interstrip position could become critical for readout pitches close to the silicon thickness. If the coupling to the read-out electrodes becomes comparable to the coupling with the back side of the device, a significant fraction of the signal is lost and this can result in a reduction of the efficiency in the floating strip region. A similar behaviour is produced when the signal is lost due to parasitic couplings as is the case when floating strips are used in very short devices (see section 8.3.4).

The plots of fig. 19 show the charge signal collected by the left and right strips versus the reconstructed interstrip position for the DSSDM 200 module. The sum of the two distributions shows a charge loss at the level of $30 \%$ in the middle of the interstrip region. The corresponding efficiency plot shows that the effect of the charge loss on the reconstruction efficiency is very modest and only affects the very central region with a deterioration from $98.0 \%$ to $95.6 \%$.

\subsubsection{Resolution}

In the CMS baseline tracker, the stereo strips are used to measure the so-called "secondary" coordinate, perpendicular to the high-precision one measured by the p-side strips, through the association between hits on the two views. In the case of the Barrel, the secondary coordinate coincides with the $z$ coordinate (along the beam axis); in the Forward the secondary coordinate will measure the radial position.

The correlation plots between the intercept of the tracks, measured by the beam telescope, and the hit position as reconstructed by the stereo strips are shown in fig. 20 for the two modules DSSDM 100 and DSSDM 200 . In plots $20 \mathrm{a}$ and 20c the $\mathrm{y}$-coordinate reconstructed from the n-side hits is the intrinsic high-resolution coordinate orthogonal to the stereo strips projected on the $\mathrm{y}$-axis, while in plots $20 \mathrm{~b}$ and plots $20 \mathrm{~d}$ an association was found with the corresponding p-side hits and the secondary coordinate, in this case the $\mathrm{x}$-coordinate, was reconstructed. The effect of the double-metal connection of the edge strips is clearly visible in plots 20a and 20c: if not taken into account, it will introduce an offset in the intrinsic coordinate equal to the detector width. This does not however cause ambiguities in the reconstruction of space points once the n-side hit is associated with a p-side hit, because the geometry of the module is such that the reconstructed point is univocally determined as is shown in fig $20 \mathrm{~b}$ and 20d.

The resolution in the intrinsic coordinate for both modules has been evaluated from the distribution of residuals computed using the centre of gravity technique already described for the p-side (figs. 21a and c ). The width of the two distributions is comparable: 34 vs $36 \mu \mathrm{m}$ as expected as a consequence of the presence of the floating strips. The plots of fig. 22 show the typical behaviour of the resolution across the interstrip region and its uniformity along the strip length in the two cases.

The resolution in the secondary coordinate is shown for DSSDM 100 and $\operatorname{DSSDM}_{200}$ in figs. $21 \mathrm{~b}$ and d: the width of the two distributions is respectively $327 \mu \mathrm{m}$ and $357 \mu \mathrm{m}$. From these values, obtained with a stereo angle of $100 \mathrm{mrad}$, we can extrapolate a resolution for the baseline CMS Double-Sided Stereo Module (200 $\mu \mathrm{m}$ read-out pitch, floating strip and a stereo angle of $60 \mathrm{mrad}$ ) of about $600 \mu \mathrm{m}$, well within the expected requirements.

Data taken with tilted detectors were used to study the resolution versus the angle of incident tracks. Fig. 23 shows the effect of the tilt angle on the resolution and on the average cluster size for the DSS module. The behaviour of the n-side with the angle is very similar to what was already described for the strips on the junction side.

\subsection{R\&D devices}

\subsubsection{The WEDGE detector}

The wedge detectors of the forward Silicon Tracker are identical to the rectangular devices of the barrel from the technological point of view, but the non-conventional geometry requires some optimization work: for example, the trapezoidal shape implies that the effective p-side strip pitch in the final modules will vary along the strips from 38 to $76 \mu \mathrm{m}$ and this feature may in principle affect the uniformity of the detector response.

We performed a preliminary study of different design options in a small wedge device whose strips on the p-side were subdivided in three groups: one based on a constant strip width of $14 \mu \mathrm{m}$, the second based on a constant 
width over pitch ratio of 0.37 and the last featuring a variable strip width from 14 to $26 \mu m$ mantaining a constant interstrip gap of $24 \mu \mathrm{m}$ along the detector.

In principle the three options imply small differences in the interstrip and coupling capacitance but we checked anyhow to see if this introduces systematic effects in the detector behaviour. For this purpose we tested one of these devices carring the three different geometries at a bias voltage of $100 \mathrm{~V}$ and in good conditions of signalto-noise ratio. Fig. 24a shows the overall charge response with a superimposed Landau fit; the corresponding signal-to-noise distribution peaks at 26.5 .

The operating conditions were clean enough to allow a careful study of the effects of the three different designs. We subdivided the tracks hitting the device according to the different regions and for each we plotted the cluster charge, the multiplicity distribution, the response function and the residual distribution. We found no significant differences for all these variables except for a slight worsening of the noise in the constant-gap region (fig. 24b).

The average performance in terms of position resolution for the p-side is shown in fig. $24 \mathrm{c}$ and is well within the expectations for the CMS forward detectors: sigma of $7 \mu \mathrm{m}$ (after subtracting the impact point extrapolation error of $5 \mu \mathrm{m}$ ) against a required $15 \mu \mathrm{m}$ resolution. Studying the resolution as a function of the coordinate along the p-side strips we observe a $10 \%$ improvement when moving from the larger to the smaller pitch region.

The WEDGE detector was a double-sided device with $60 \mu \mathrm{m}$ wide strips on the back side, orthogonal to the central p-side strip, at a pitch of $125 \mu \mathrm{m}$. A kapton fan-out circuit was used to connect the n-side strips to the electronics: 128 strips were read-out at $125 \mu \mathrm{m}$ pitch and 128 were read-out every $250 \mu \mathrm{m}$ leaving one floating strip between two electrodes. The residual distribution for the $125 \mu \mathrm{m}$ read-out zone is shown in fig. $24 \mathrm{~d}$. It appears as a uniform distribution with a FWHM of $80 \mu \mathrm{m}$, corresponding to a sigma of about $23 \mu \mathrm{m}$. The same plot for the $250 \mu \mathrm{m}$ read-out pitch region with floating strip gives a similar resolution.

The strip length on the n-side of the WEDGE device was very short (ranging from 4.8 to $6.3 \mathrm{~mm}$ ): this implies a weak interstrip coupling which heavily affects the charge response when a floating strip is left between two read-out electrodes. This problem is treated in detail in Section 8.3.4.

\subsubsection{The DSODM and the BABY detectors}

The possibility of using the double-metal technology to obtain high-resolution information also in the reconstruction of the second coordinate has been evaluated looking at the performance of DSODM and BABY detectors. In these two devices the n-side strips are orthogonal to the p-side strips and the signals are brought to the read-out electronics through a connection on a second metal layer separated from metal-1 by a $4 \mu m$ thick oxide.

This technique avoids the use of external kapton fan-out circuits but introduces additional parasitic capacitance which may affect the performance of the device. We tested this technique in two different detectors: the DSODM where strips on the n-side run at a $100 \mu \mathrm{m}$ pitch for a length of $19.2 \mathrm{~mm}$ while the metal-2 lines are about $61 \mathrm{~mm}$ long on a $50 \mu \mathrm{m}$ pitch; the BABY device where strips of the same length on the n-side $(19.2 \mathrm{~mm})$ are drawn at a $50 \mu \mathrm{m}$ pitch and the metal-2 lines are $8 \mathrm{~mm}$ long on a $100 \mu \mathrm{m}$ pitch.

Fig. 25a shows the charge response of the n-side of the DSODM device; there is a good correlation between the charge collected on the two sides (fig. 25c).

The n-side cluster shape (shown in fig. 25b) has the typical behaviour expected for standard devices. The residual distribution for $100 \mu \mathrm{m}$ pitch is shown in fig. 25d. The width of this distribution is $58 \mu m$ FWHM (corresponding to a sigma of about $25 \mu \mathrm{m}$ ). The p-side resolution of this detector is $11 \mu \mathrm{m}$.

The performance that could be achieved with this technique has been investigated looking at the data of the BABY device where a read-out pitch of $50 \mu \mathrm{m}$ is used for the n-side too. The detector response appears to be within expectations in terms of charge, signal-to-noise distribution, and $\eta$ function (fig. 26). The residual distribution after correcting for non-linearity in the $\eta$ function is shown in fig. $26 \mathrm{~d}$ : the sigma of the residual distribution is $6.9 \mu \mathrm{m}$ and by subtracting the track extrapolation error we obtain a detector resolution of $4.7 \mu \mathrm{m}$.

The p-side of the BABY detector, where we used the floating strip technique, was affected by the same problem already mentioned for the n-side of the WEDGE: very short strips, weakly coupled to the read-out electrodes, producing distortions in the charge response. A detailed description of the problem is given in Section 8.3.4.

\subsubsection{The PAD detector}

An alternative approach to the use of stereo strips for the second coordinate has been tested with devices having the $\mathrm{n}$-side subdivided into pads [21]. The n-side is organized in rows of 32 pads, $6.4 \mathrm{~mm}$ wide and $2 \mathrm{~mm}$ high, whose 
signals are routed to the read-out electronics sitting on the edge of the detector with a separate kapton circuit, glued on top of the device. We expect that this detector, without increasing the number of channels, could provide a position resolution similar to the stereo detectors with the pattern recognition problem simplified by the use of true space points.

The most probable signal-to-noise measured for this device was 7.7; a preliminary investigation of the reasons for this low value points out the combined effects of non-negligible capacitance contributions from the kapton circuit and of large common mode fluctuations due to imperfect filtering of the read-out circuitry.

From the residual distribution shown in fig. 27a we measure a position resolution of about $600 \mu \mathrm{m}$ in the perpendicular coordinate for this device. The tracking potential of the device can be appreciated from fig. 27b, which shows the correlation between the hits reconstructed by the pad device and the track position as predicted by the beam telescope.

\subsubsection{Charge response in presence of weakly coupled floating strips}

The use of floating strips, although convenient in terms of reduction of the number of read-out channels, may introduce distortions in the detector response. The mechanism of charge sharing between the floating strips and the read-out electrodes may be critical when the coupling is too weak. The charge collected on the floating strips induces signals on all surrounding electrodes in accordance to the coupling capacitances; when the coupling with the read-out electrodes is comparable with the coupling to passive elements, a significant fraction of the charge is lost and the uniformity in the detector response is affected. This is the case, for instance, when the strips are very short. Some parasitic capacitance to the guard-ring and to the back of the detector is always present; usually, for a strip length of several centimeters, these couplings are much lower than the coupling to the read-out electrodes, which increases linearly with the strip length (at the rate of 1-1.4 pF/cm). When very short strips are used, the coupling to the read-out electrodes may be lower than $1 p F$ and every parasitic coupling of the same order of magnitude will cause substantial charge loss. A similar case may appear when the interstrip gap is too wide: for read-out pitches above $200 \mu \mathrm{m}$ the coupling to the back becomes important and a large fraction of the charge is lost when tracks hit the region of the floating strip. This problem was observed in the data of the WEDGE detector on the $\mathrm{n}$-side and of the BABY detector on the $\mathrm{p}$-side.

Figs. 28a and 28b show the anomalies seen in the n-side cluster signal and in the correlation plot for the WEDGE. These plots show a clear double-peaked distribution that can be explained looking at the cluster multiplicity. The charge response of the $\mathrm{n}$-side for single-strip clusters (fig. 28c) shows a good Landau distribution; if we plot the charge collected by multi-strip clusters we obtain another good Landau distribution (fig. 28d) but the peak value in this case is much lower (40\% lower) than the previous one.

Since the charge sharing mechanism is strongly dependent on the hit position we explain this result with a charge loss for tracks hitting the intermediate region between the read-out electrodes and therefore seen by the floating strip.

The very short n-strips ( 4.8 to $6.3 \mathrm{~mm})$ and the large read-out pitch $(250 \mu \mathrm{m})$ imply a weak coupling of the floating electrodes to the read-out strips. It should be noticed that this result has no impact on the position resolution of the detector but will affect the efficiency and uniformity of response of the device.

A similar behaviour was noticed for the p-side of the BABY detector giving us further evidence that the mechanism is independent of the read-out side. Fig. 29a shows the distribution of the charge collected on the p-side and again the double-peaked distribution can be resolved by plotting separately the charge collected in single- (fig. 29b) and multi-strip clusters (fig. 29c). In this case too, the coupling of the floating electrodes to the read-out strips is very weak, due to the very short strip length $(8.2 \mathrm{~mm})$, and comparable with the parasitic coupling to the guard ring elements.

The charge loss in the central region has been studied with the BABY by plotting the $\eta$ distribution (fig. 29d) which shows a very low central peak in correspondence of the floating electrode, and by studying the most probable signal on the left and right strip versus the interstrip position. The plots in fig. 30 compare the distributions of this variable for the p-side and for the n-side. From the distribution we can estimate a signal loss up to $60 \%$ in the middle of the interstrip gap on the p-side.

The effect of this charge loss on the efficiency of the device has been carefully investigated. The overall efficiency of the p-side, excluding a few dead or disconnected strips, is about $97 \%$ while the corresponding value for the $\mathrm{n}$-side exceeds $99.6 \%$. The inefficiency on the p-side is uniformly spread over the detector, being mostly due to clusters with signal below threshold caused by the previously decribed mechanism. 
For a better understanding of the problem the device was also tested in laboratory using a laser diode. Infrared light pulses having a wavelength of $940 \mathrm{~nm}$ and collimated to within $20 \mu \mathrm{m}$ were shot across the interstrip region and the corresponding signals were digitized. Fig. 31 shows the result of the scan transverse to the p-side strips. The average cluster signal is plotted as a function of the laser spot position. A substantial reduction of the cluster charge appears exactly at the position of the floating strip, thus confirming the result obtained with charged particles.

\section{Summary and Conclusions}

The results obtained so far can be considered promising in terms of detector performance.

The measured resolution of all tested detectors is well within the requirements set for the CMS Silicon Tracker.

The value of 26:1 for the signal to noise ratio measured on the SSFS module appears quite satisfactory. As a matter of fact, the final single-sided modules will differ from the module tested mainly in the use of more sophisticated read-out electronics. With respect to the performance of the PreMux128, the final front-end chip is expected to have an ENC worse by a factor 1.4, mostly due to the modified filtering of signals provided by the deconvolution circuit, while the contribution of the analog pipeline and of the optical link are expected to be negligible. One must also take into account the effect of irradiation on detectors and read-out electronics. Nevertheless, even considering higher leakage currents, increases of the interstrip capacitance, and some charge loss on the p-side due to the lower field after inversion, we still have a large margin before reaching an average signal to noise ratio of 10:1, which is considered our lower limit for safe operation after 10 years of high-luminosity operation at the LHC.

We have demonstrated that high voltage operation, which will be needed to keep full efficiency after irradiation, is feasible with single sided detectors without a significant noise increase. Our study is currently limited to $200 \mathrm{~V}$ and single sided detectors, but preliminary measurements suggest that the range can be extended safely at least up to $300 \mathrm{~V}$. A thorough study is foreseen in forthcoming beam tests for single- and double-sided devices before and after irradiation.

We have proved that the double metal technology can be used to solve of the problem of dead regions at the edges of double sided stereo devices. The performances of stereo devices featuring $100 \mu \mathrm{m}$ and $200 \mu \mathrm{m}$ read-out pitch with and without floating strip, have been carefully investigated. Although the overall efficiency does seem satisfactory, the charge loss affecting the floating strip region requires an optimization of the detector design. Further study is needed to improve the signal to noise ratio of the n-side with particular care to the filtering of the detector bias lines.

We have obtained very promising preliminary results on the performance of wedge detectors similar to those that will be used in the forward disks.

Lastly, several R\&D devices have been investigated including PAD detectors and high resolution double-sided devices.

Acknowledgements

We wish to thank all people whose support has been essential for this work, in particular the technical staff of the INFN laboratories, the colleagues of other CMS institutions who provided crucial elements of the read-out electronics, and all people involved in the setting up and running of the two beam tests at CERN.

\section{References}

[1] The Compact Muon Solenoid, “Technical Proposal”, CERN/LHCC 94-38 LHCC/P1, Dec 15, 1994

[2] P. Weiss et al., “Wafer-Scale Technology for Double-Sided Silicon Microstrip Particle Detectors", The $7^{\text {th }}$ International Conference on Solid-State Sensors and Actuators

[3] G. Tonelli et al., Nucl. Instrum. and Meth. A377 (1996) 422

[4] A. Holmes-Siedle et al., NIM A339 (1994) 511

[5] E. Catacchini et al. "Wedge silicon detectors for the inner tracking system of CMS", presented at the $6^{t} h$ Topical Seminar on "Experimental Apparatus for Particle Physics and Astrophysics" in S.Miniato 1996, to be published in Nucl. Phys. B Proceedings Supplement

[6] RD20 Collaboration, “RD20 Status Report 1995”, CERN-LHCC/96-2 
[7] RD20 Collaboration, "RD20 Status report", CERN/DRDC 94-39

[8] J. Matheson et al., “ Radiation Damage Studies of Field Plate and p-stop n-side Silicon Microstrip Detectors", accepted for publication by NIM

[9] M. French, L. Jones, P. Murray, P. Seller, M. Raymond, G. Hall, "APV5-RH: a 128 Channel Radiation Hard Pipeline Chip for LHC Tracker Applications", Proceedings of 1st Workshop on Electronics for LHC Experiments, CERN/LHCC/95-56.

[10] M. Raymond and L. Jones, "RD20 PreShape32 User Manual"

[11] L. L. Jones, "PreMux128 User Manual”

[12] L. Celano et al., "A High Resolution Beam Telescope Built with Double Sided silicon Strip Detectors", accepted for publication by NIM

[13] IPN Lyon, S. Electronique, "BUFFER pour detecteurs SILICIUM" and "BASE carte HT".

[14] RD5 Collaboration, “Status Report of the RD5 Experiment”, CERN/DRDC 93-49

[15] C. Albajar et al., NIM A364 (1995) 473

[16] RD5 Collaboration, “RD5 '93 Trigger System”, RD5 TN/93-14, Nov 21993

[17] W. Dulinksi et al., "VME Flash ADC multi-purpose module”, Draft, VFLAM-CRN/Lepsi 1992.

[18] O. Adriani et al., "A data acquisition system for silicon microstrip detectors", Dipartimento di Fisica e INFN Firenze Preprint DFF 237/11/95.

[19] “CMSIM User Manual”

[20] “Simulation of VDET”, ALEPH Internal Note 92-052, 1992

[21] R. Wheadon et al., "Double-sided silicon detectors using $n$-side pad readout for the CMS silicon inner tracker", 1995 IEEE Nuclear Science Symposium and Medical Imaging Conference Record Oct 21-28 1995, San Francisco, Guest Editor Patricia A. Moonier, Volume 1 


\begin{tabular}{|l|l|l|l|l|l|l|l|l|l|l|}
\hline Module & side & SS & SSFS & DSS & DSODM & BABY & DSSDM $_{100}$ & DSSDM $_{200}$ & PAD & WEDGE \\
\hline Signal/Noise & $p$ & 27. & 26.1 & 8.2 & 23.2 & $15.9^{*}$ & 14.9 & 13.4 & - & 26.5 \\
\hline Signal/Noise & $n$ & - & - & 9.5 & 17. & 21. & 8.4 & 11. & 7.7 & $38.4^{*}$ \\
\hline
\end{tabular}

Table 3: Most probable value of the signal to noise ratio for all tested detectors at nominal conditions. Figures marked with an asterisk were obtained averaging the results from separate distributions for single- and multi-strip clusters.

\begin{tabular}{|l|l|l|l|l|l|l|l|l|l|l|}
\hline Module & side & SS & SSFS & DSS & DSODM & BABY & DSSDM $_{100}$ & DSSDM $_{200}$ & PAD & WEDGE \\
\hline Resolution & $p$ & 12. & 11. & 14. & 11. & 16. & 12. & 12. & - & 7. \\
\hline Resolution & $n$ & - & - & 275. & 17. & 6. & $\begin{array}{c}325 \cdot a^{a)} \\
32 .\end{array}$ & $\begin{array}{c}357 . .^{a)} \\
35 .\end{array}$ & 600. & 23. \\
\hline
\end{tabular}

Table 4: Spatial resolution in $\mu m$ for all tested detectors. Superscript a) refers to the resolution in the secondary coordinate (orthogonal to the one measured by the p-side), while b) refers to the resolution in the intrinsic $n$-side coordinate. 


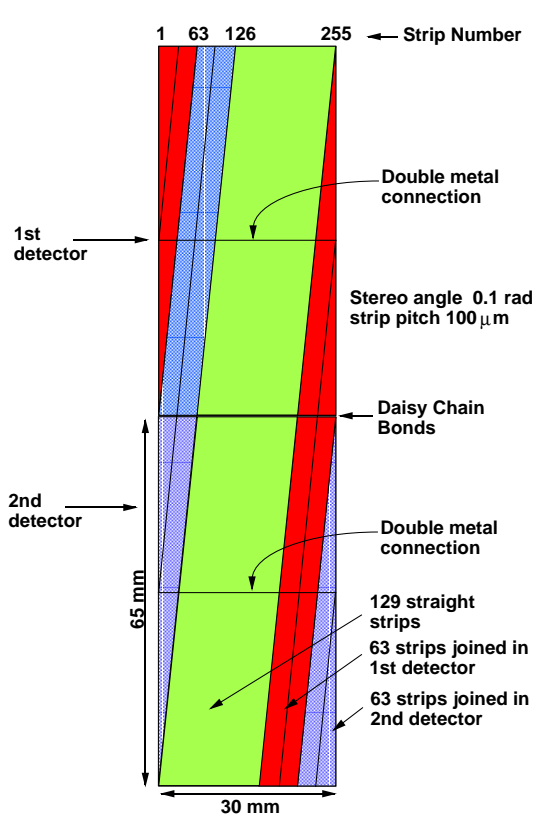

Figure 1: Schematic description of two DSSDM detectors assembled in a module.

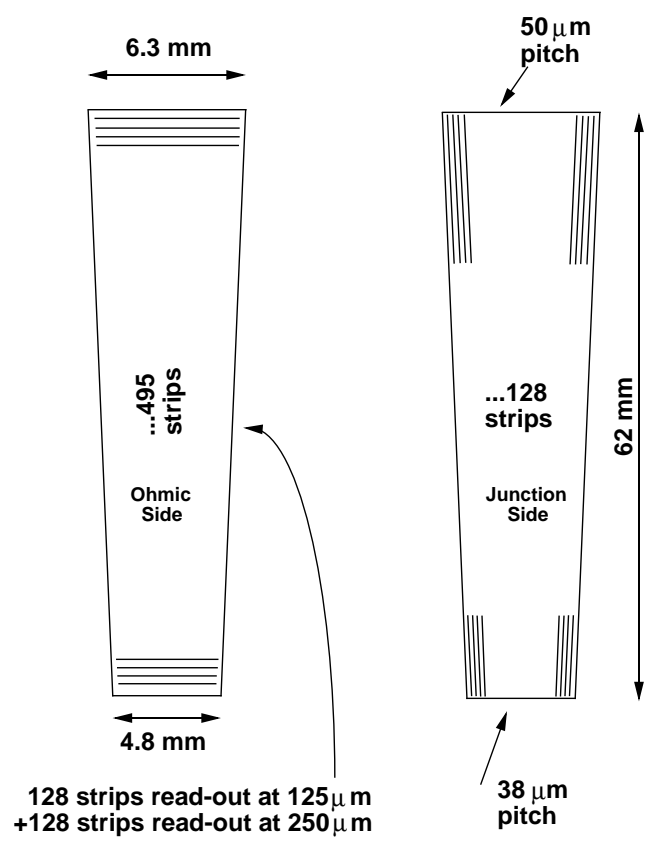

Figure 2: Wedge detector schematic description. 


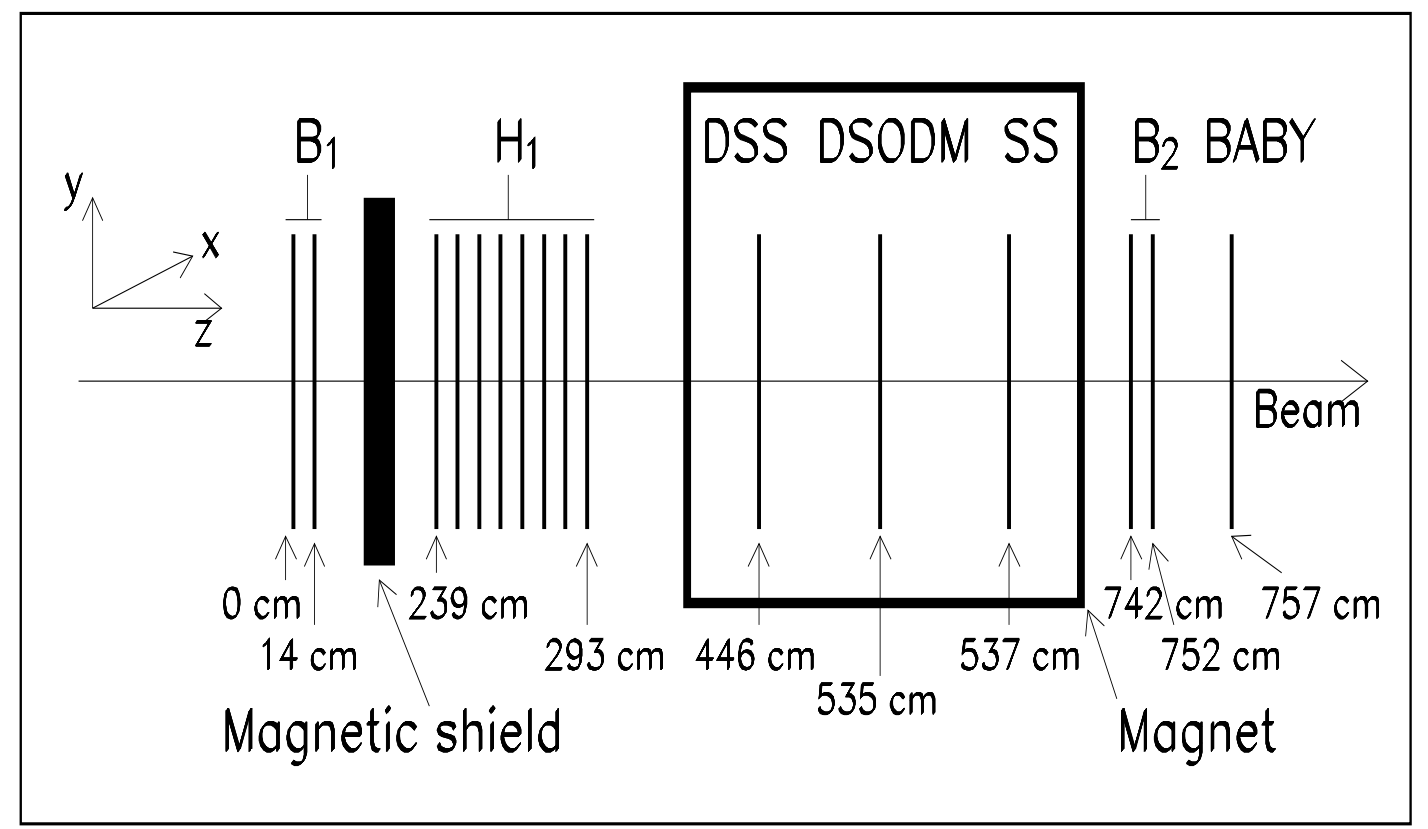

Figure 3: July 95 set-up

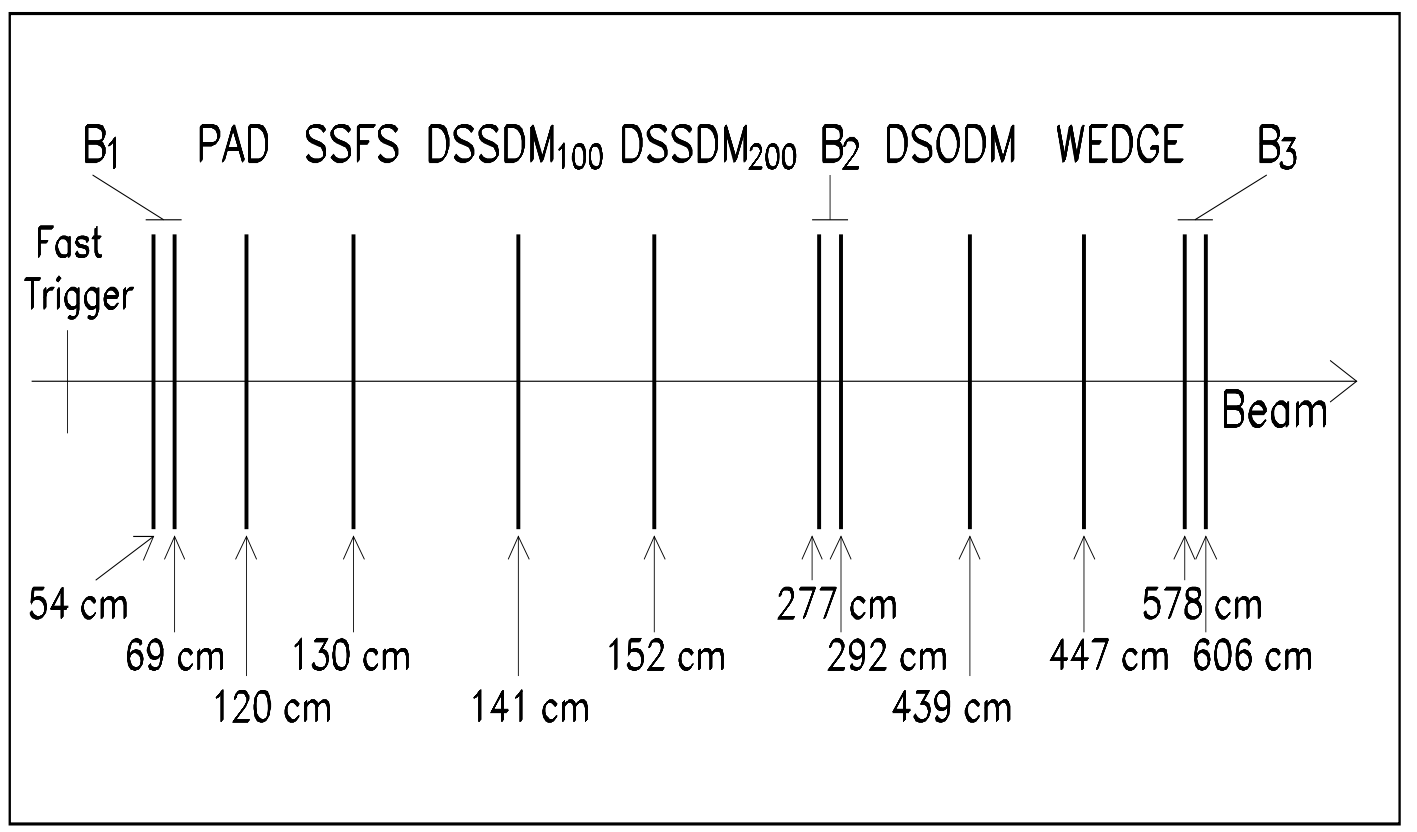

Figure 4: September 95 set-up 

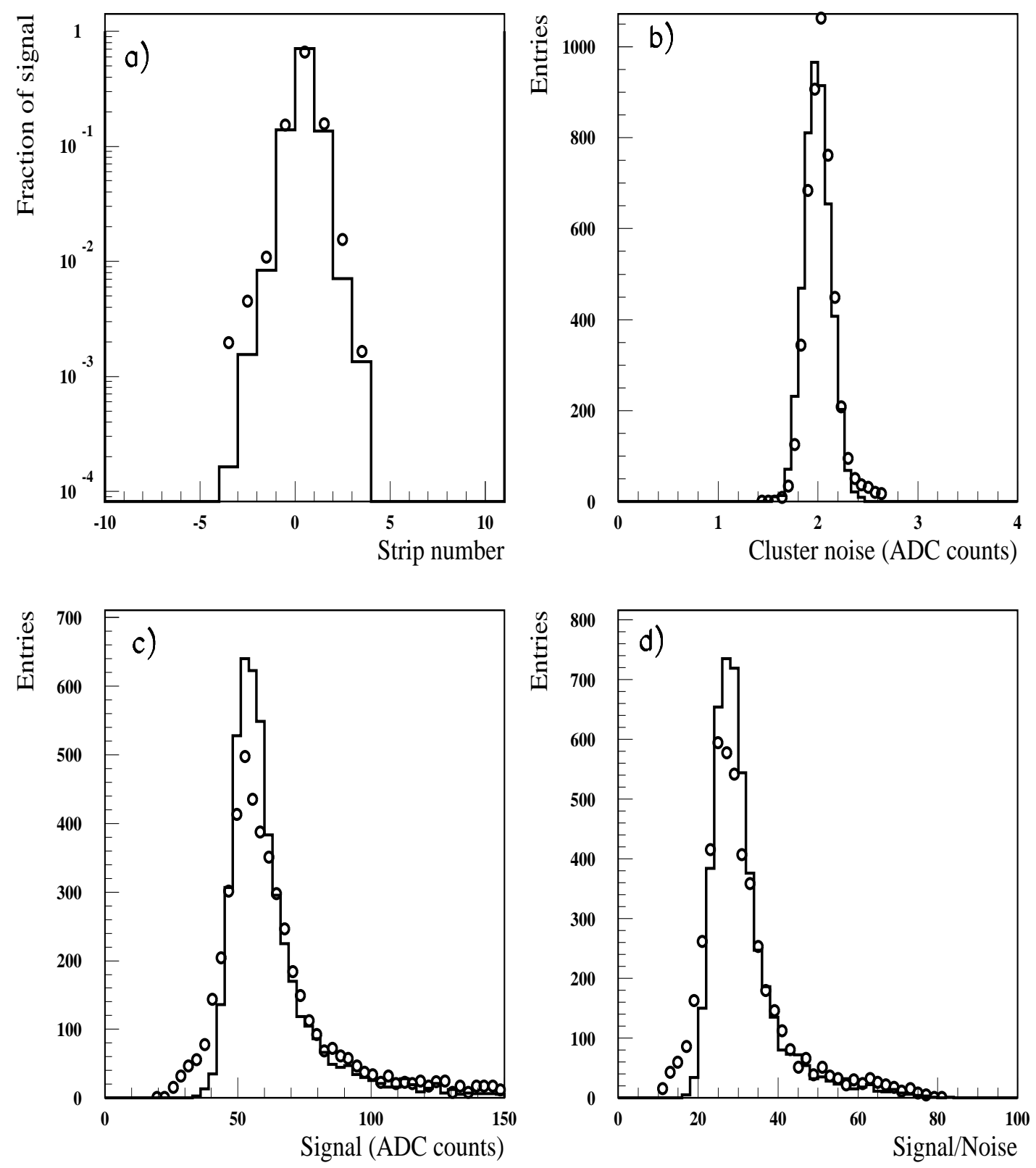

Figure 5: Comparison between MC (full line) and real data (dots): a) Cluster shape; b) Cluster Noise; c) Cluster Signal; d) Signal to Noise ratio. 

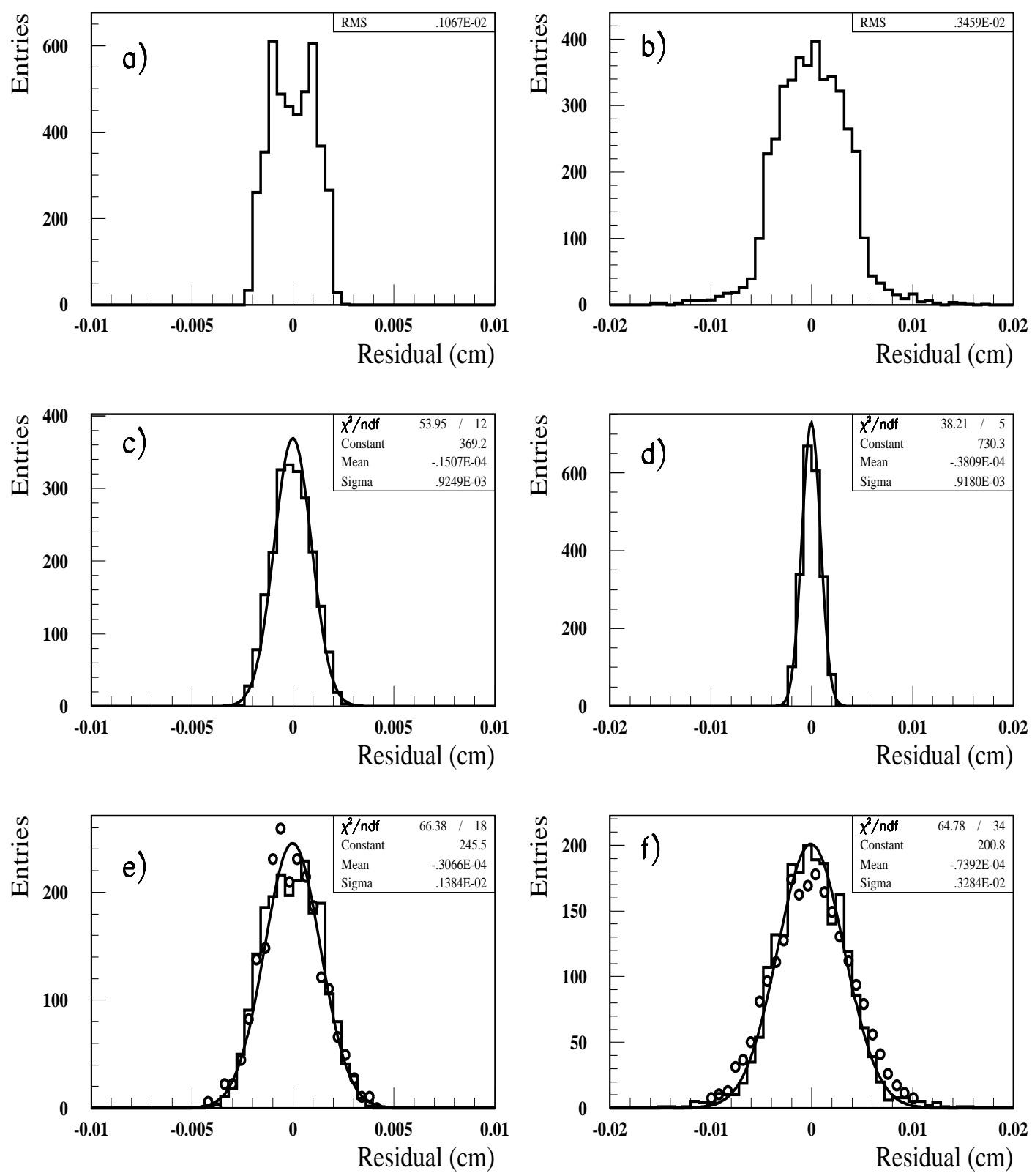

Figure 6: Plots on the first column refer to SSFS while those on the second column refer to DSSDM 200 : a) and b) Simulated detector intrinsic resolution; c) and d) Error on track extrapolation point as evaluated by the MC; e) and f) Comparison between MC (histogram and fit) and real data (dots) distributions of residuals for the two detectors. 

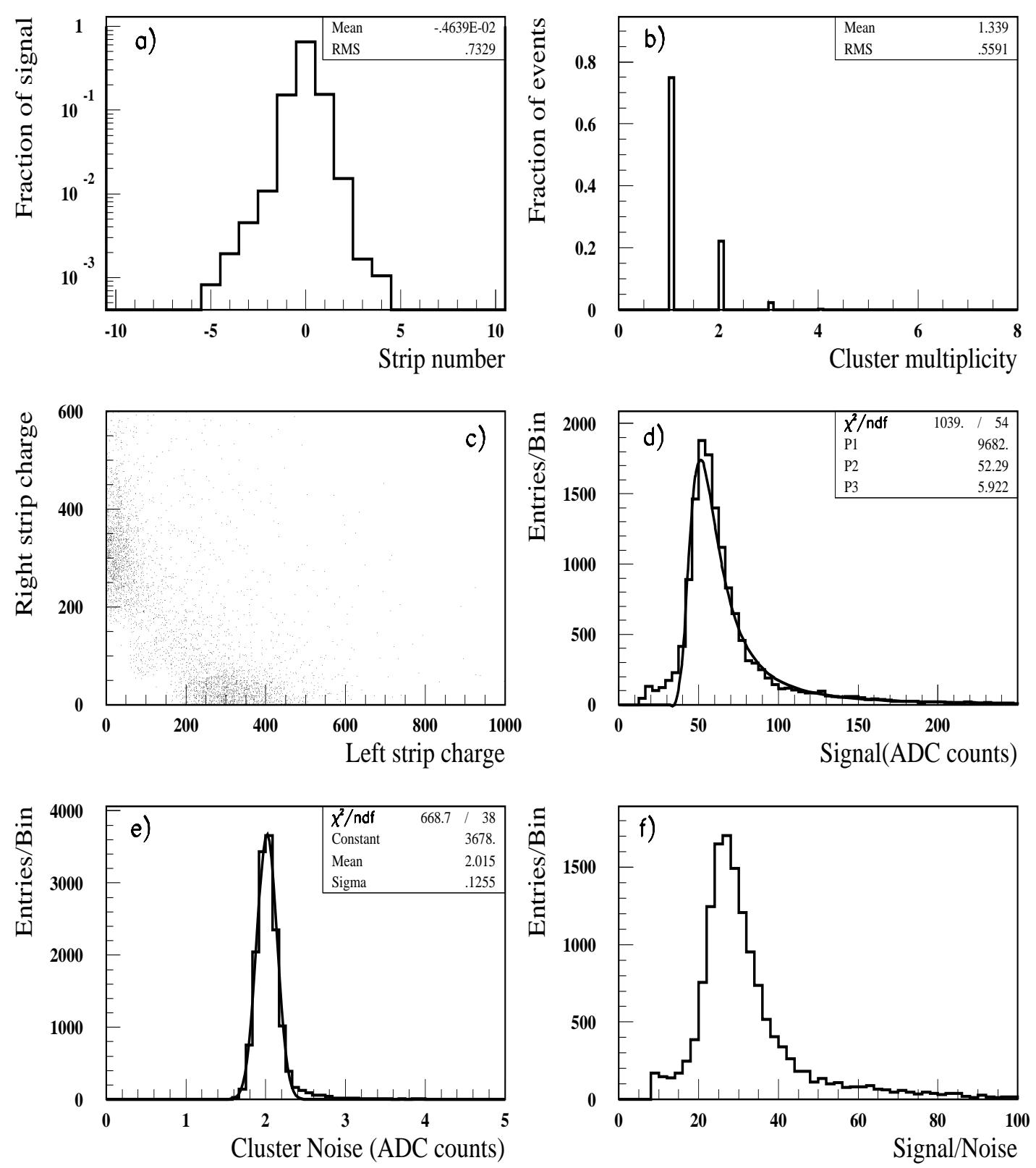

Figure 7: Data for a p-side 50 per cluster; c) Charge collected on left and right strips; d) Cluster Signal; e) Cluster Noise; f) Signal to Noise ratio. 

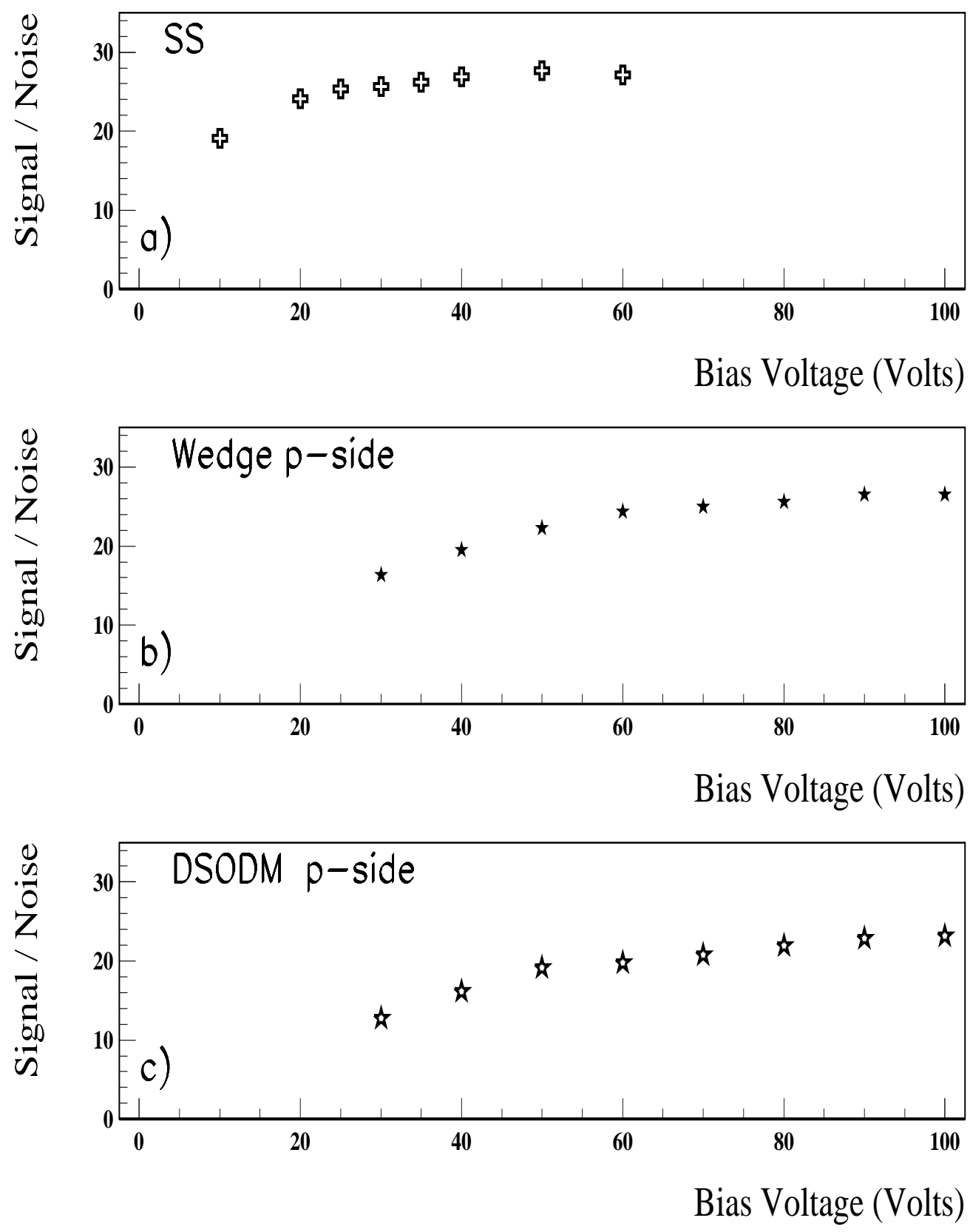

Figure 8: Signal to Noise ratio versus the applied voltage for: a) SS, b) WEDGE p-side, c) DSODM p-side. 


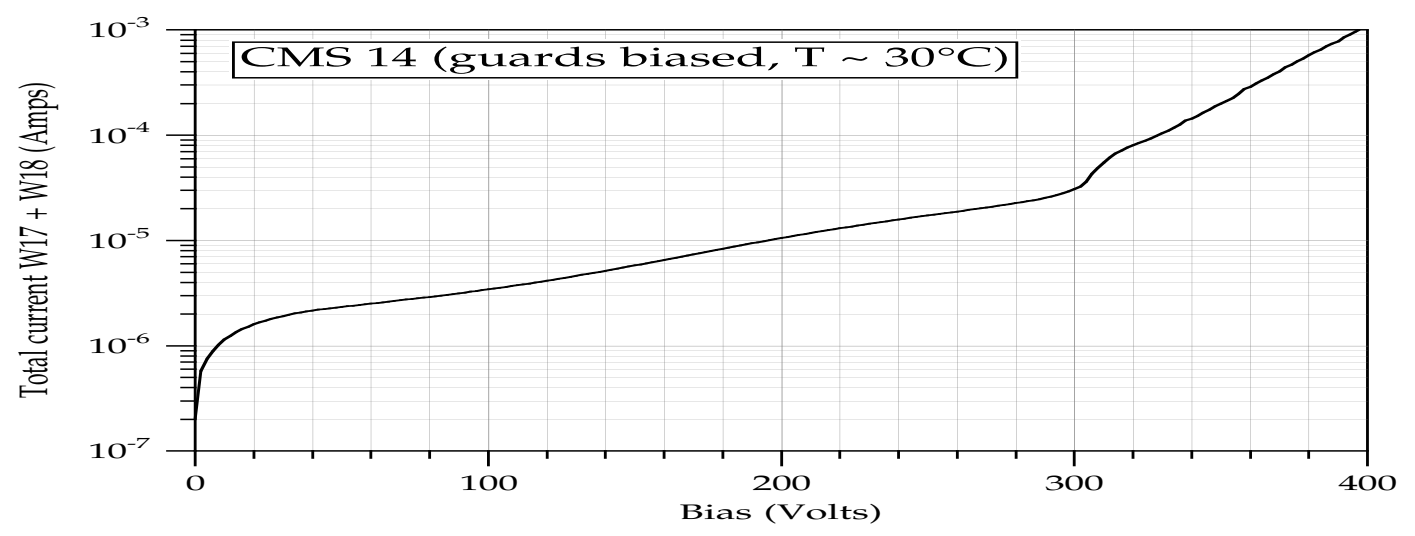

Figure 9: Laboratory measurement of the I-V curve for SSFS
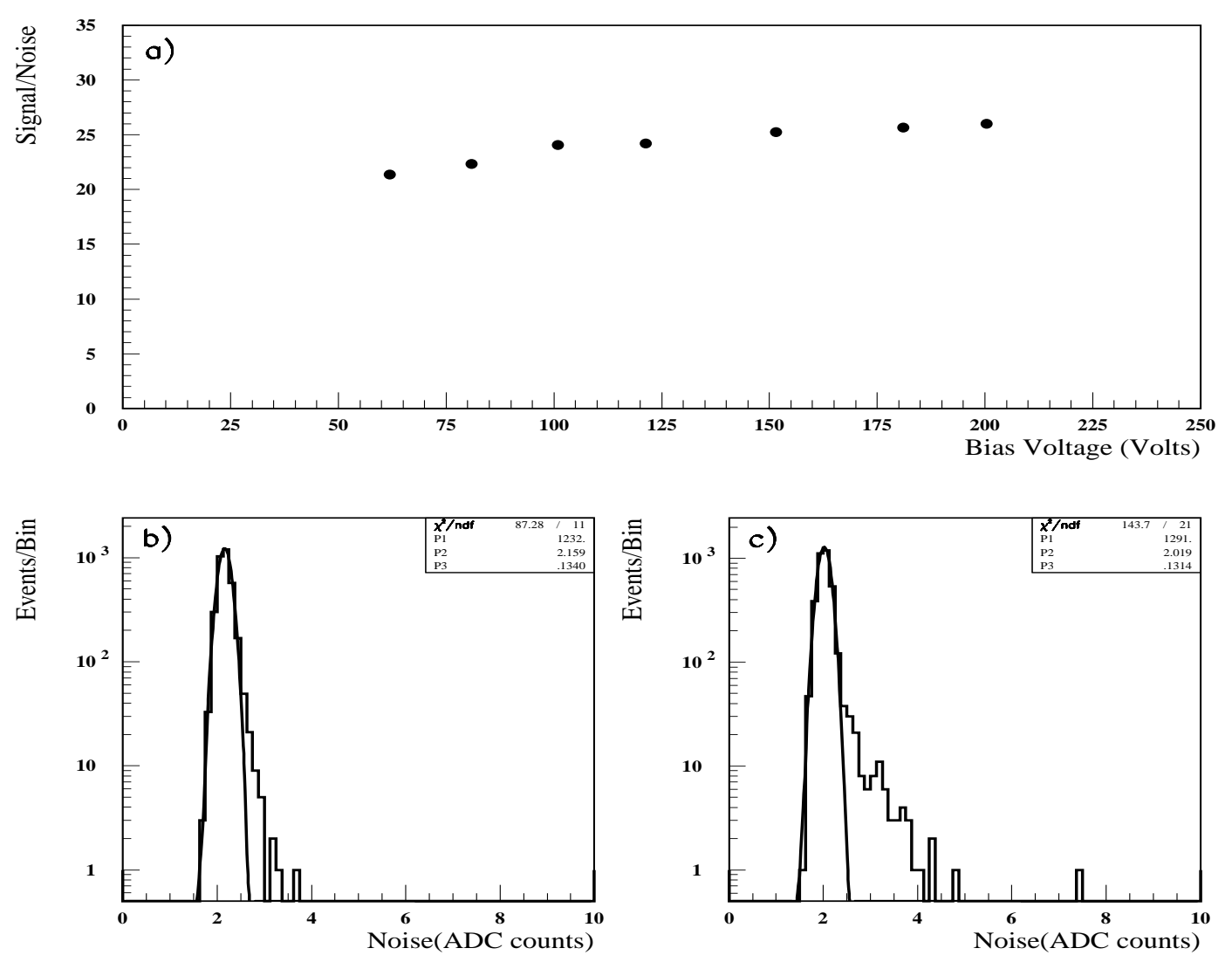

Figure 10: a) Signal to Noise ratio versus the applied voltage for SSFS; b) Cluster Noise distribution at $V_{\text {bias }}=70 \mathrm{~V}$; c) Cluster Noise distribution at $V_{\text {bias }}=200 \mathrm{~V}$. 

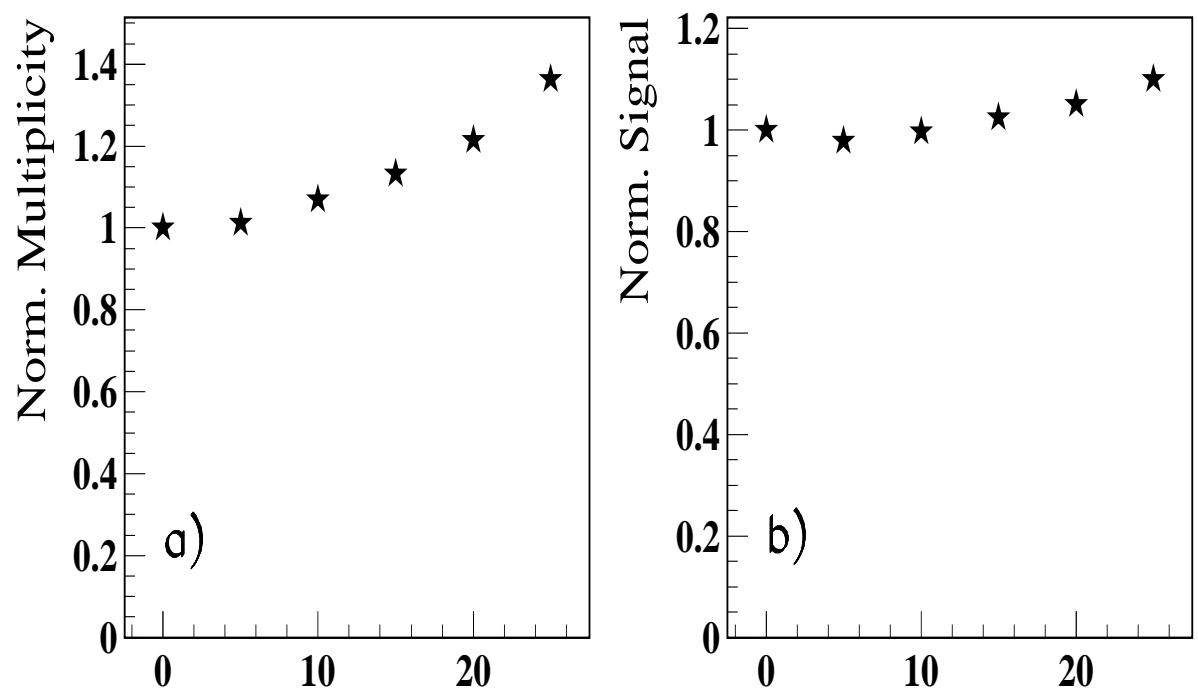

Tilt Angle (Deg.)
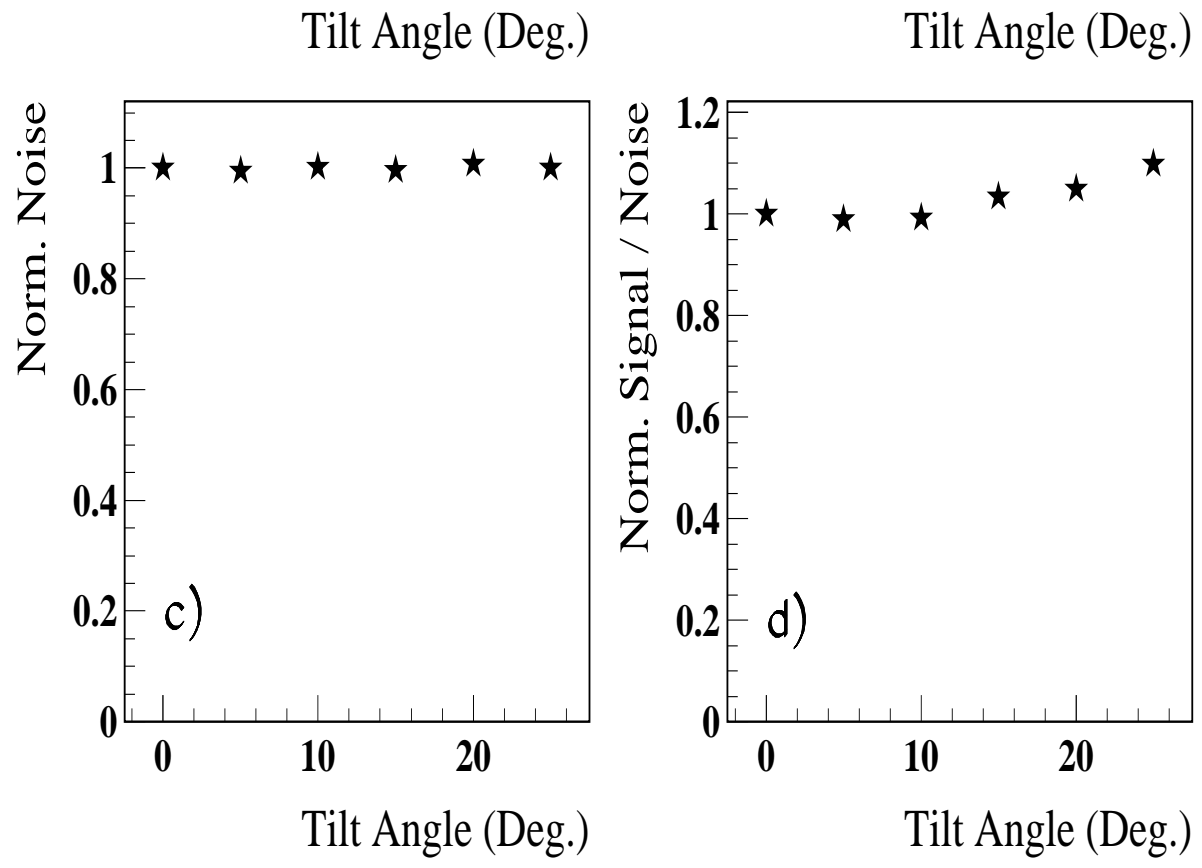

Figure 11: Scan in the tilt angle for the p-side of a DSODM detector; all points are normalized to the orthogonal incidence values: a) Number of strips in cluster, b) Cluster Signal, c) Cluster Noise, d) Signal to Noise ratio. 

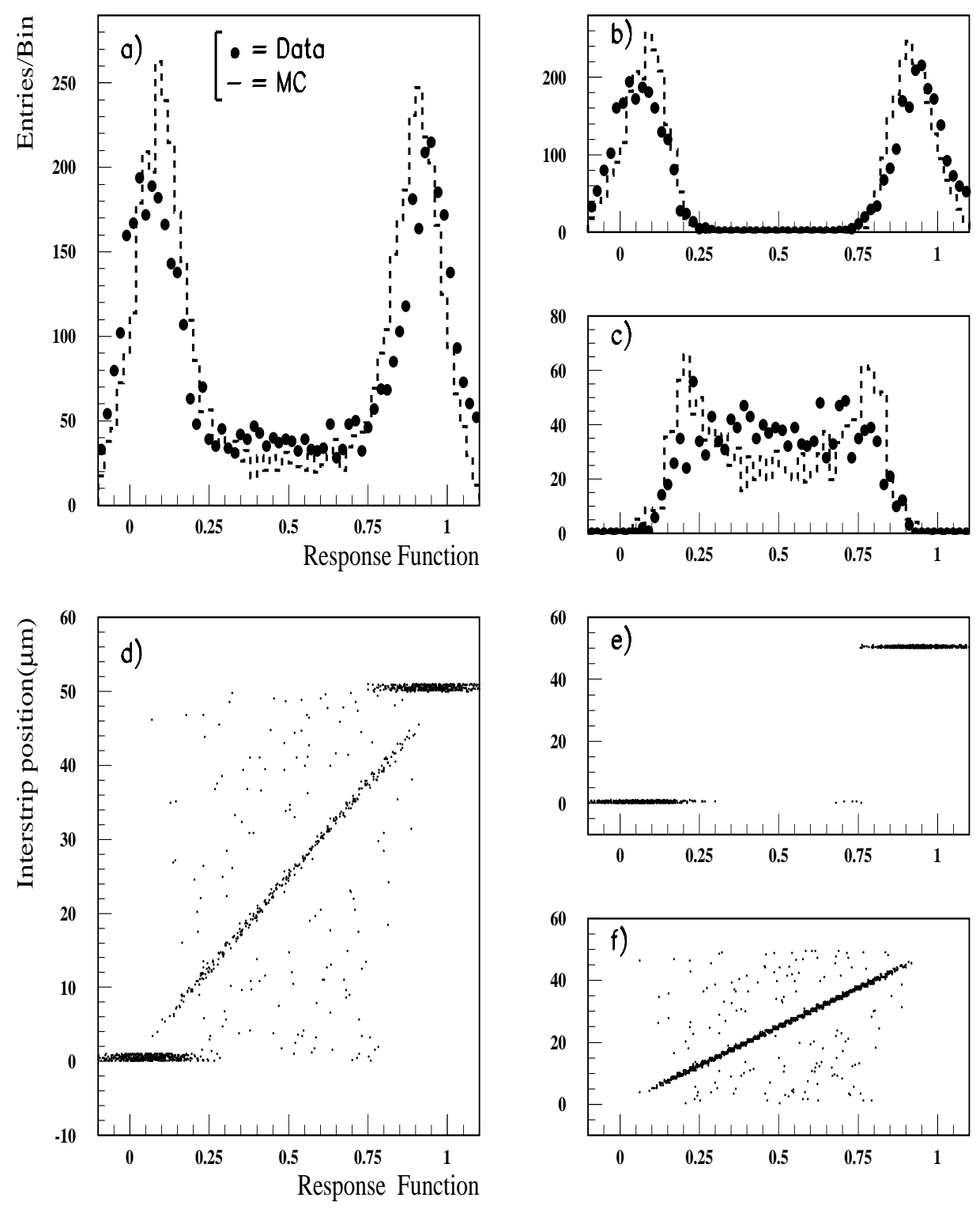

Figure 12: a) Response Function for DSS p-side with MC simulation superimposed; b) and c) same as a) for single- and multi-strip clusters respectively; d) Interstrip position versus Response Function for real events; e) and f) same as d) for single- and multi-strip clusters respectively. 

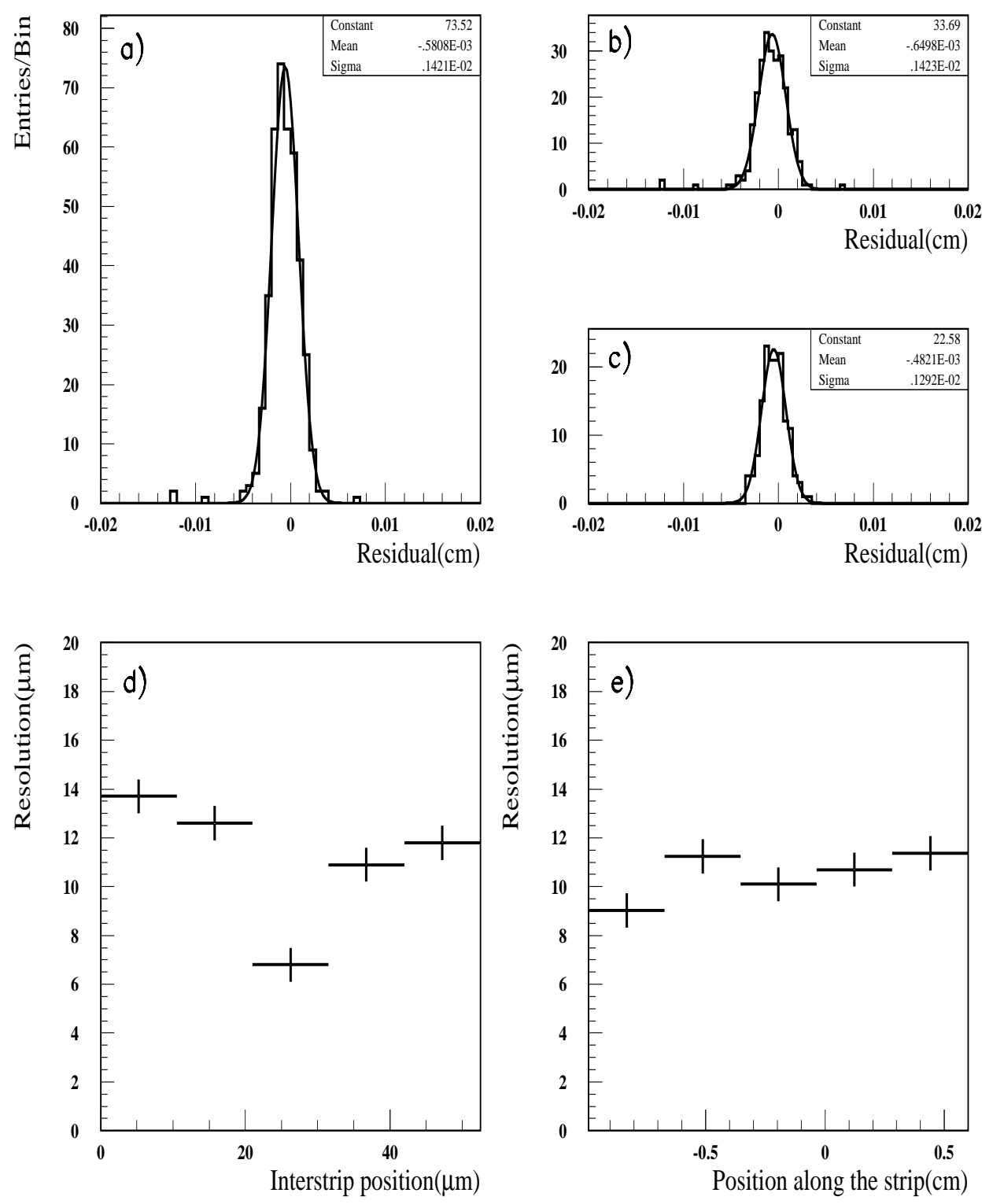

Figure 13: a) Residuals for a p-side 50 $\mu$ m pitch detector (SSFS); b) and c) same as a) referring to single- and multi-strip clusters respectively; d) Resolution versus the interstrip position; e) Resolution versus the position along the strip. 

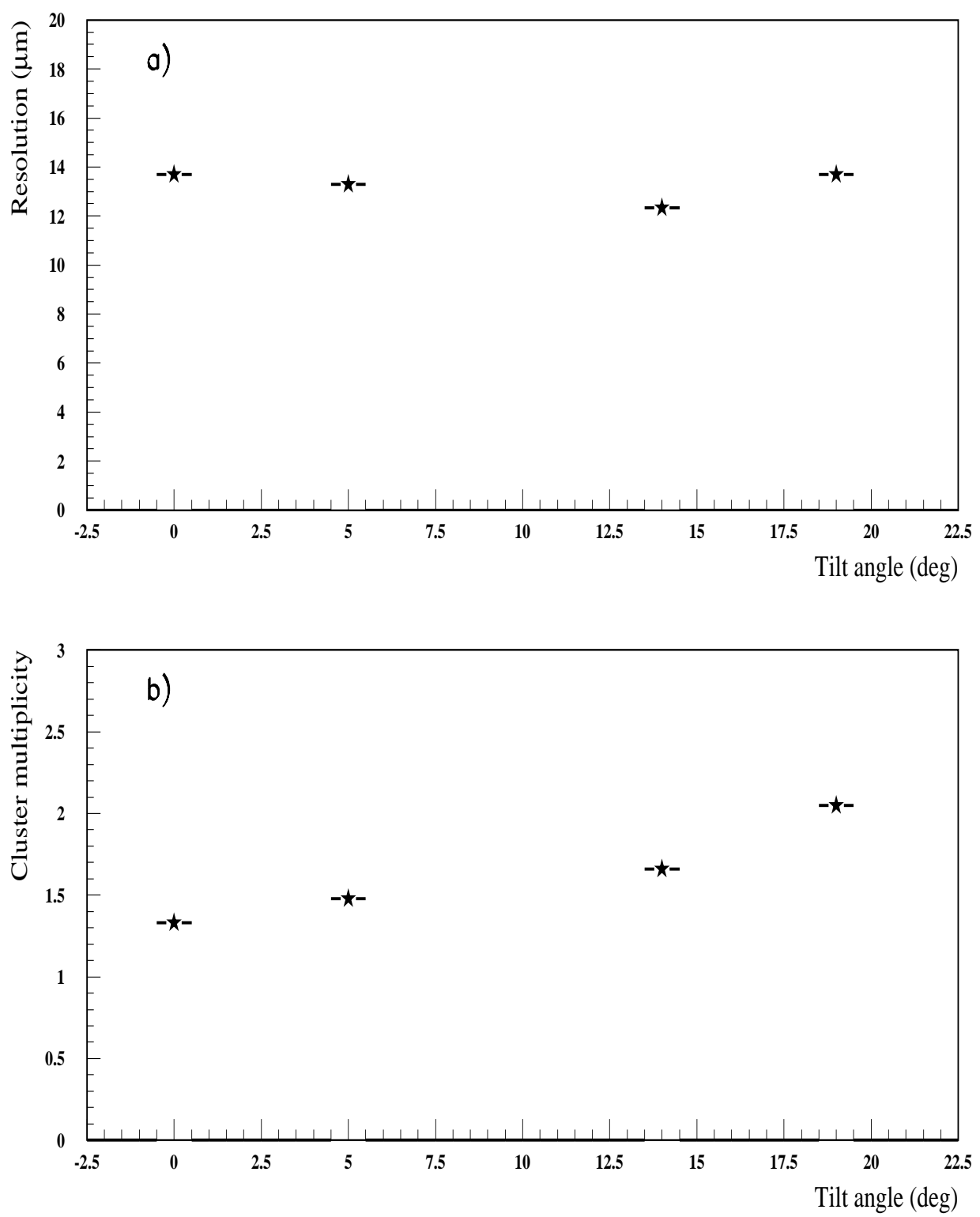

Figure 14: Scan in the tilt angle for the p-side of the DSS detector: a) Resolution; b) Number of strips per cluster. 

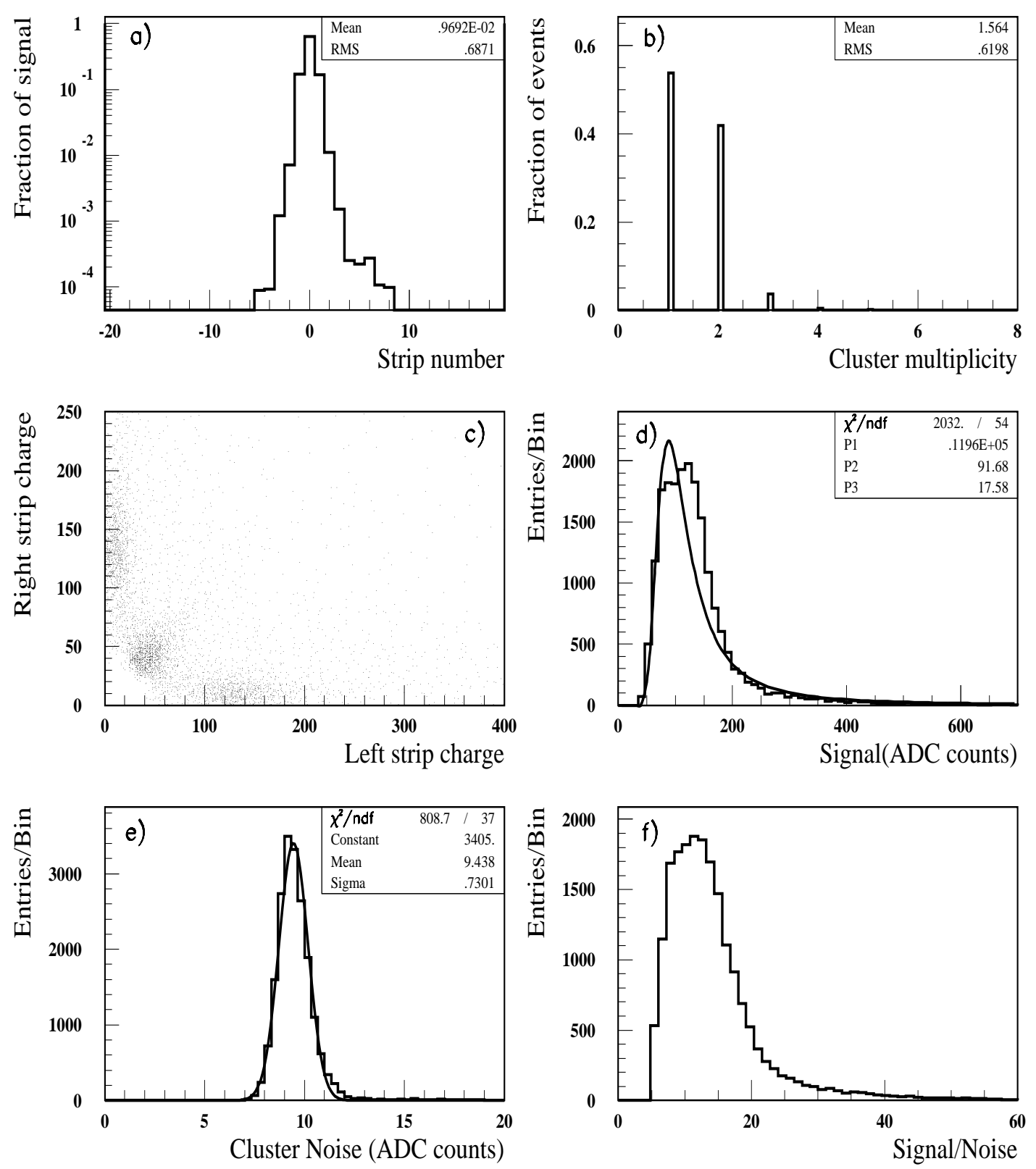

Figure 15: Data for the n-side of DSSDM 200: a) Cluster shape; b) Number of strips per cluster; c) Charge collected on left and right strips; d) Cluster Signal; e) Cluster Noise; f) Signal to Noise ratio. 

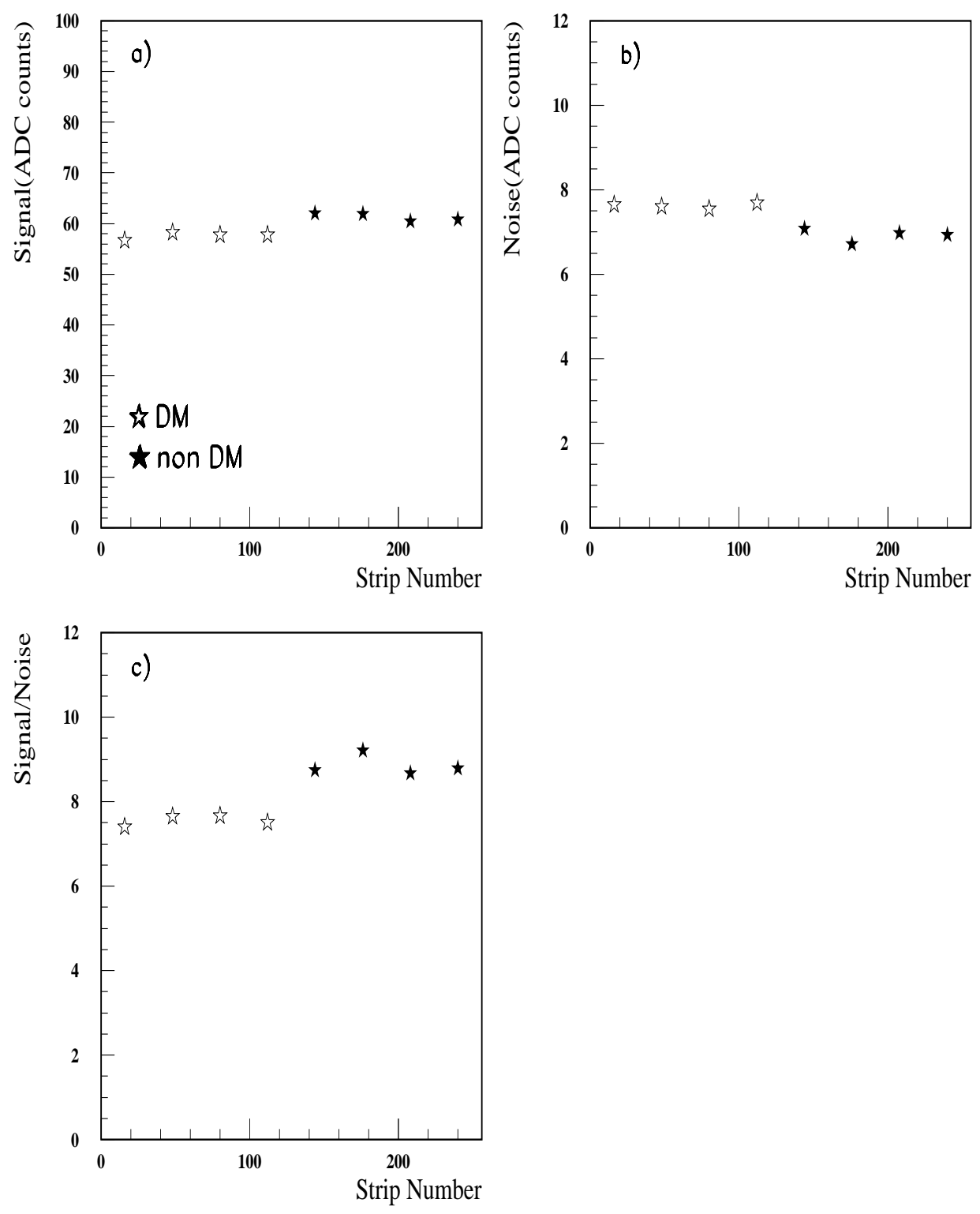

Figure 16: Data for the n-side of (DSSDM $\left.M_{100}\right)$. Average values are evaluated in bins of 32 strips. DM strips go from 1 to 128 while non-DM strips go from 129 to 256: a) Most probable value of the Cluster Signal; b) Average Cluster Noise; c) Average Signal to Noise ratio. 


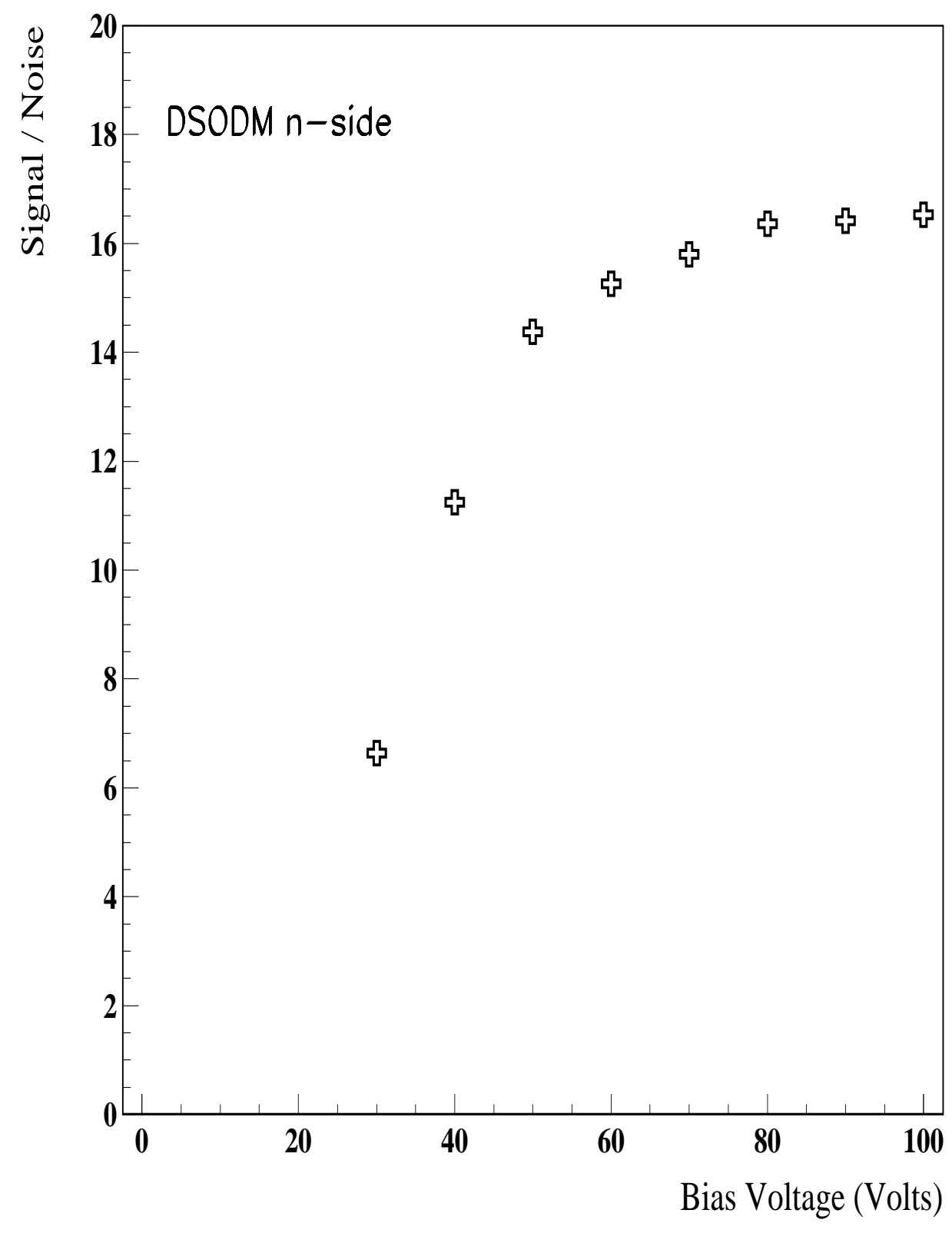

Figure 17: Signal to Noise ratio for the n-side of DSODM as a function of Bias Voltage. 

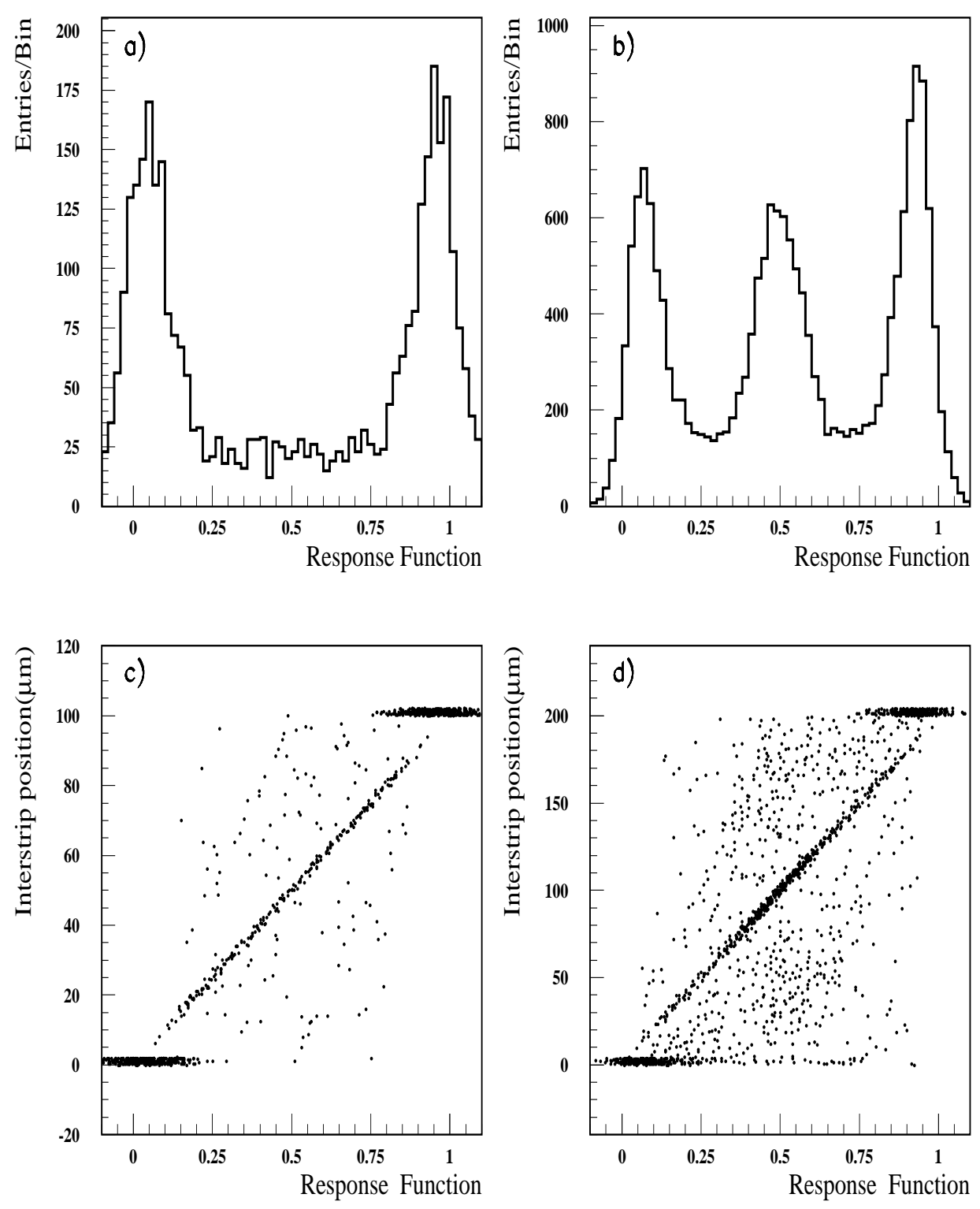

Figure 18: a) Response Function for the n-side of $\operatorname{DSSDM}_{100}$; b) same as a) for the n-side of DSSDM ${ }_{200}$; c) Interstrip position versus the Response Function for the n-side of $D_{S S D M} M_{100}$; d) same as c) for the n-side of $\operatorname{DSSDM}_{200}$. 

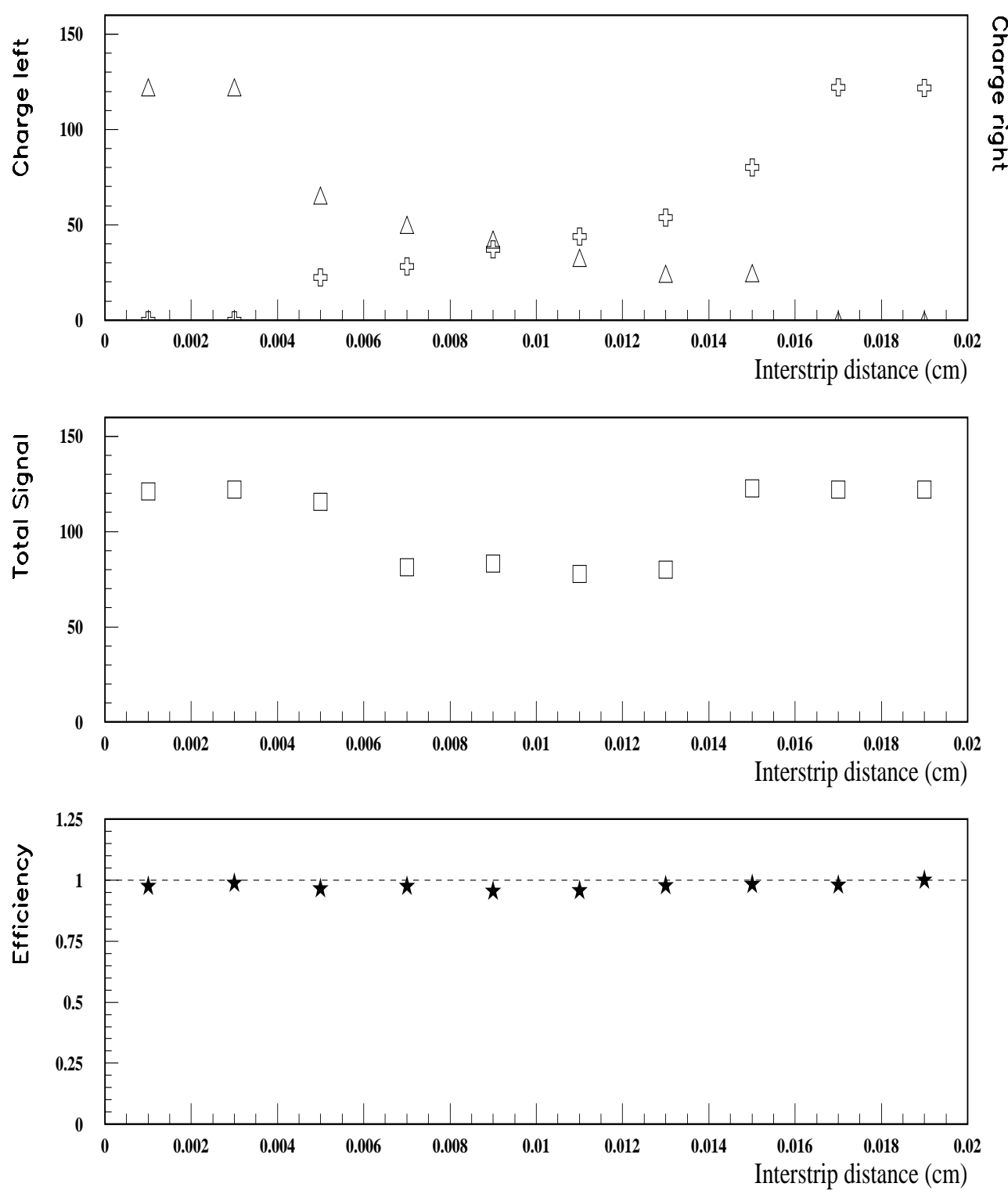

Figure 19: Data from the n-side of $D_{S S D M} M_{200}$ : a) Charge collected on the left and right strips (ADC counts); b) Total Signal; c) Efficiency. All variables are plotted versus the reconstructed position modulo the strip pitch. 

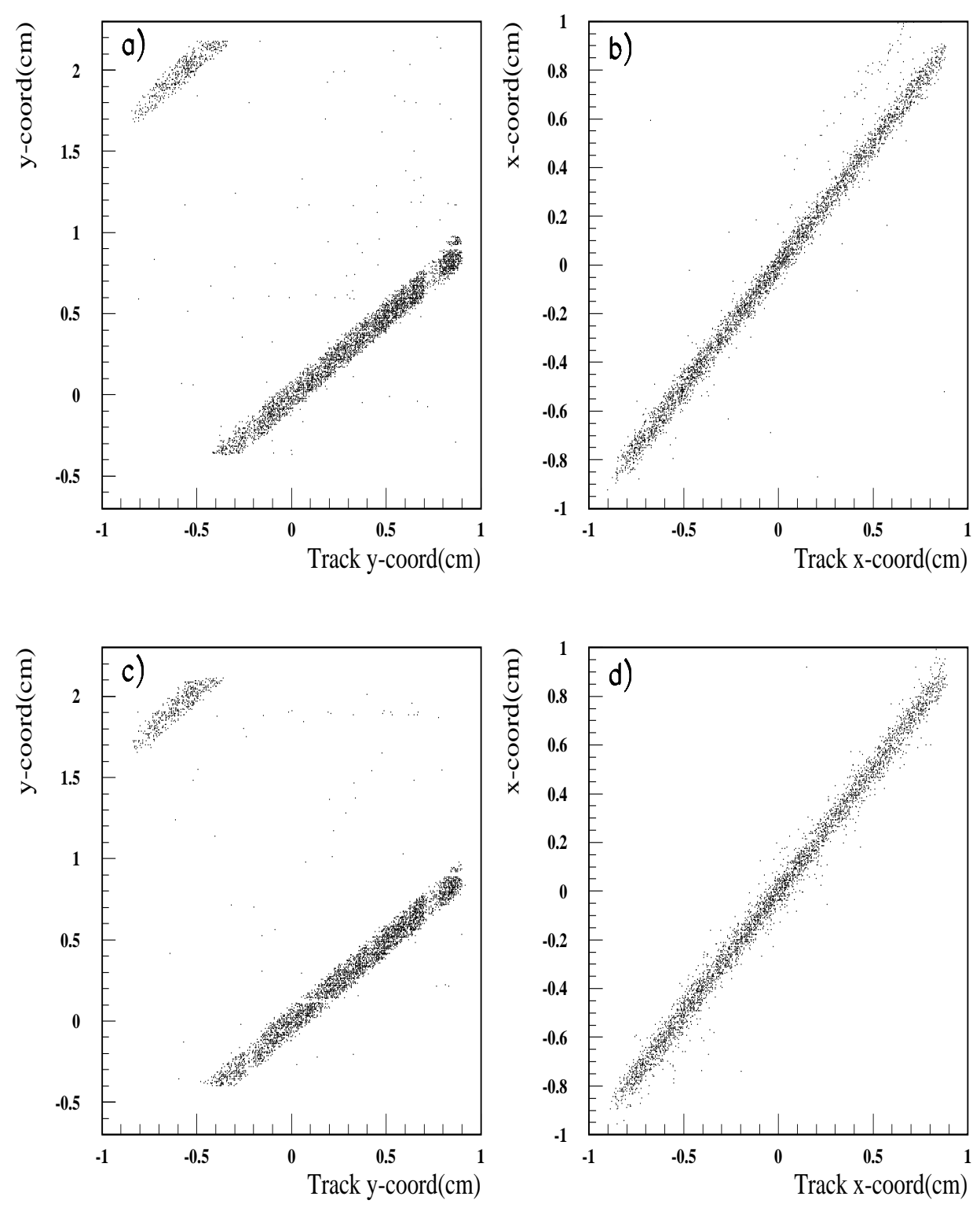

Figure 20: Correlation between the interpolated track position and the point locally reconstructed by the stereo strips: a) and b) refer to $D_{S S D M_{100}}$, c) and d) refer to $\operatorname{DSSDM}_{200}$. The $y$-coord is the coordinate almost orthogonal to the stereo strips; the $x$-coord is the 'secondary' coordinate. 

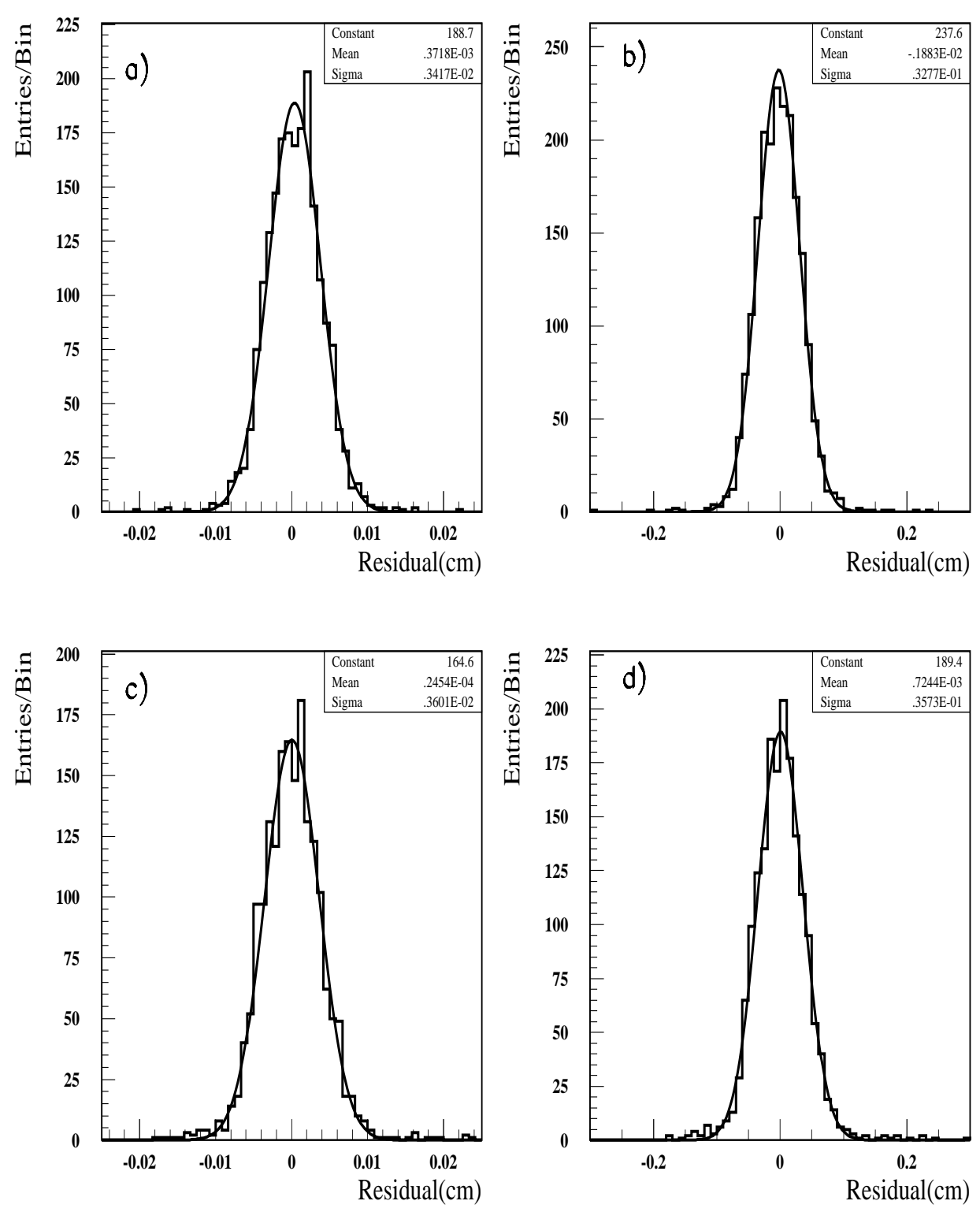

Figure 21: a) Residuals in the intrinsic coordinate for the n-side of $D S S D M_{100}$; b) Residuals in the secondary coordinate for the n-side of DSSDM 100 ; c) same as a) for $D_{S S D M_{200}}$; d) same as b) for DSSDM 200 . 

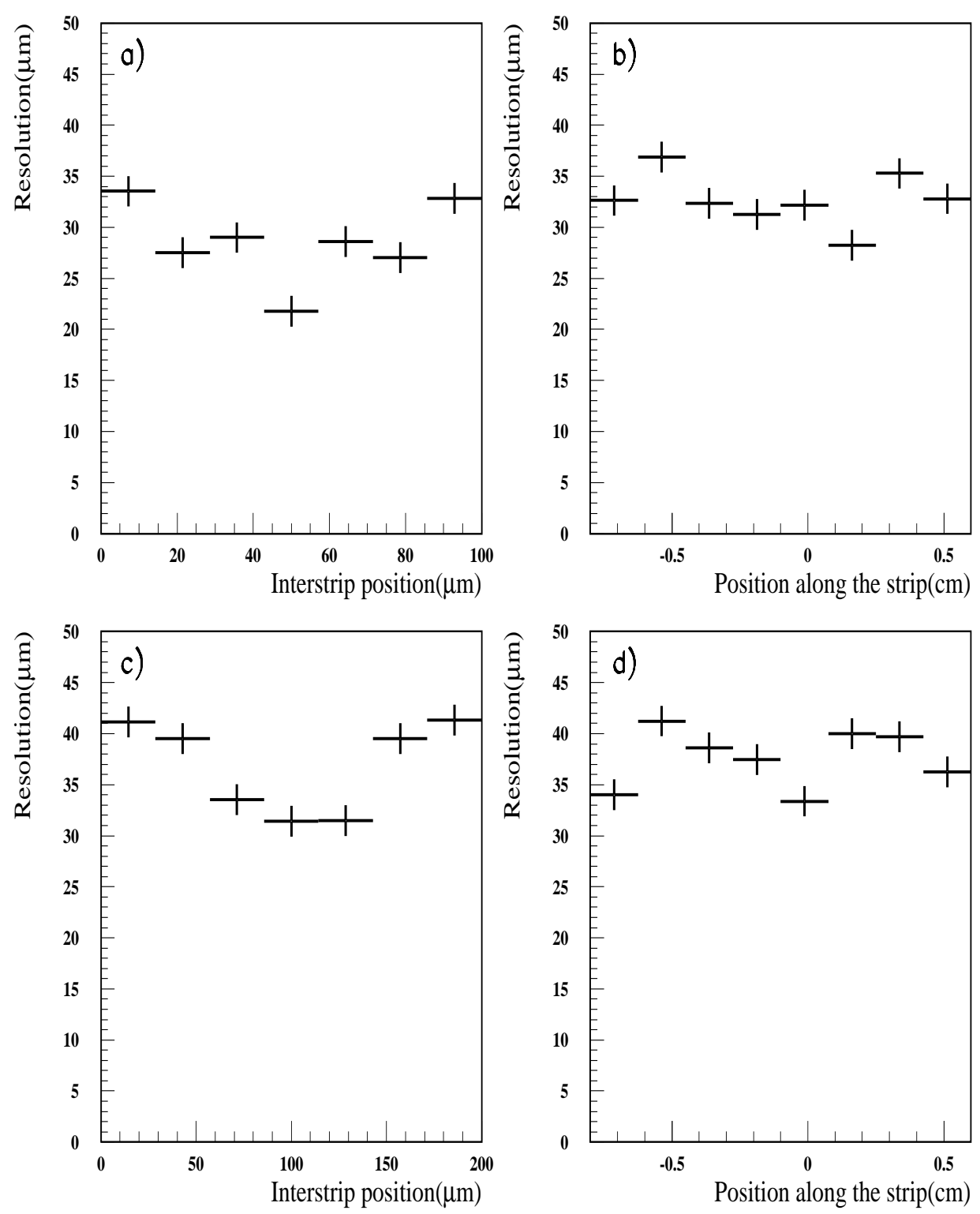

Figure 22: Resolutions in the intrinsic coordinate: a) Resolution versus the interstrip position for the n-side of $D S S D M_{100}$; b) Resolution versus the position along the strip for $D S S D M_{100}$; c) same as a) for $D_{S S D M_{200}}$; d) same as b) for $\mathrm{DSSDM}_{200}$. 


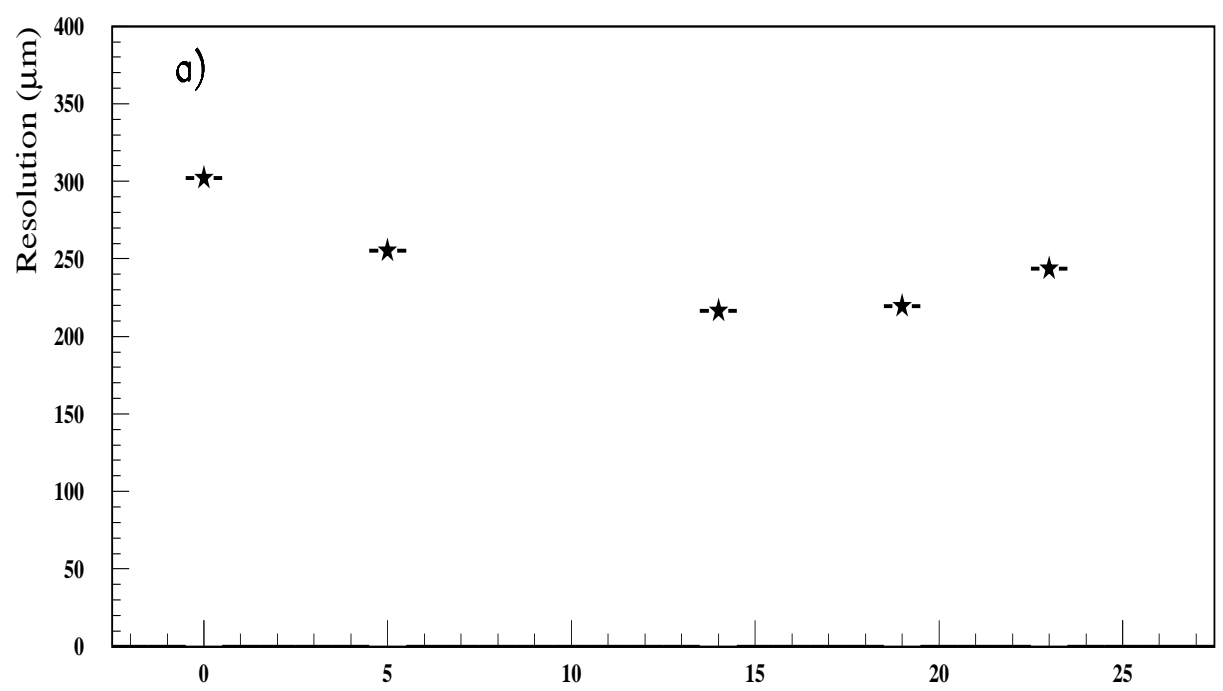

Tilt angle (deg)

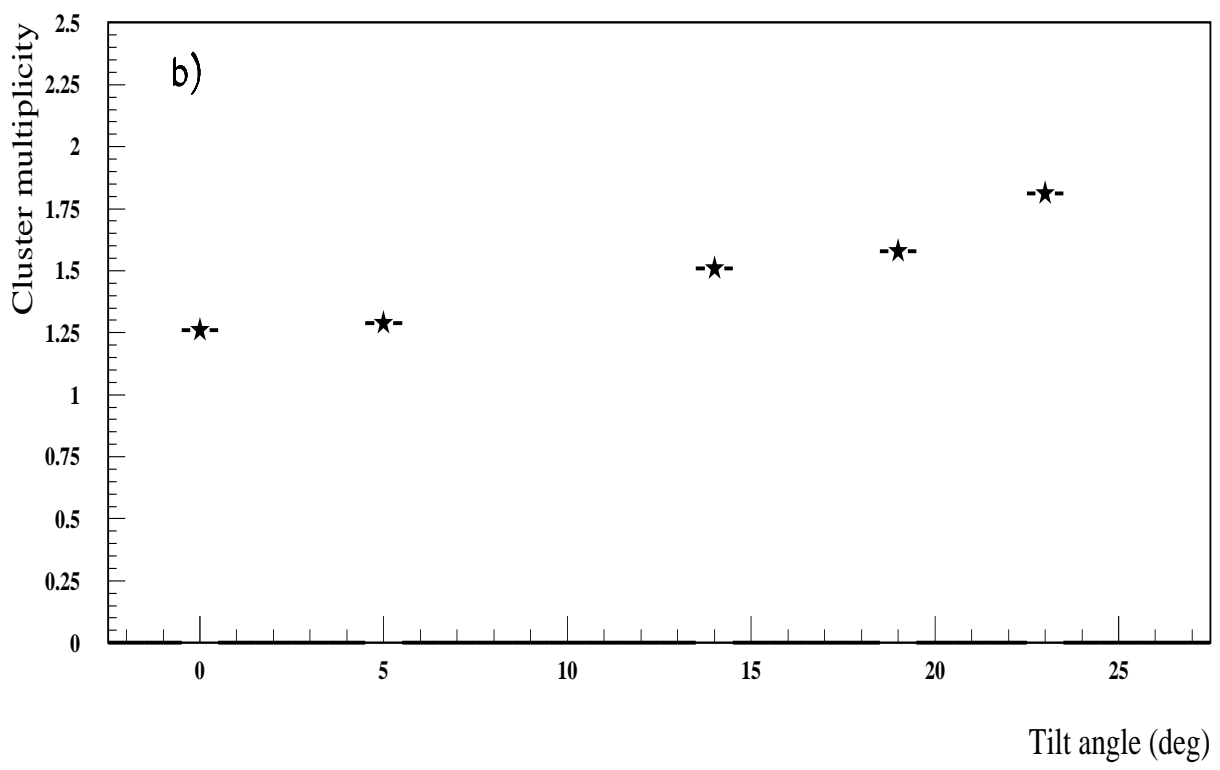

Figure 23: Scan in the tilt angle for the n-side of DSS: a) Resolution; b) Number of strips per cluster. 

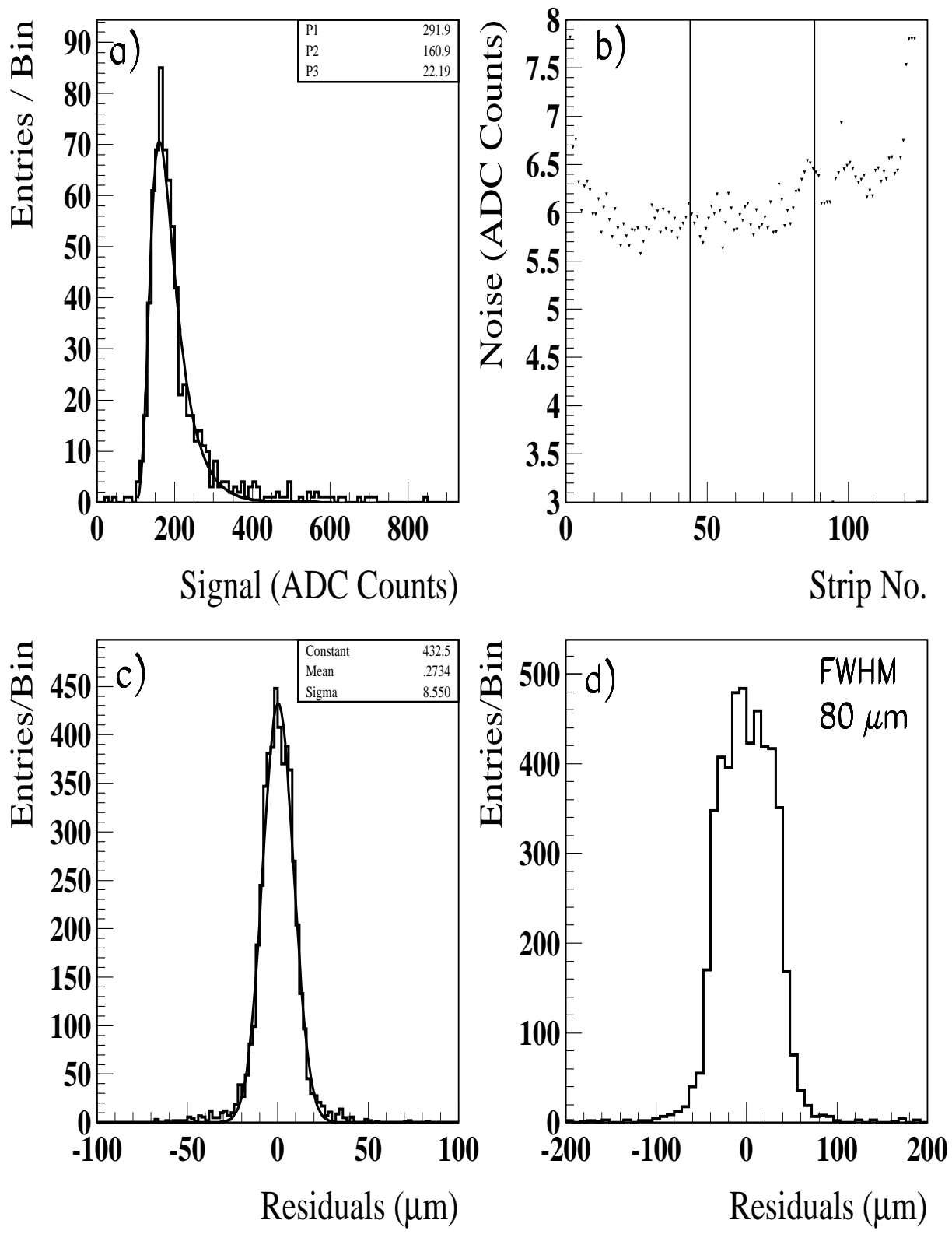

Figure 24: Data for WEDGE: a) p-side Cluster Signal; b) p-side strip Noise for the three different regions (see text); c) p-side Residual distribution; d) n-side Residual distribution. 

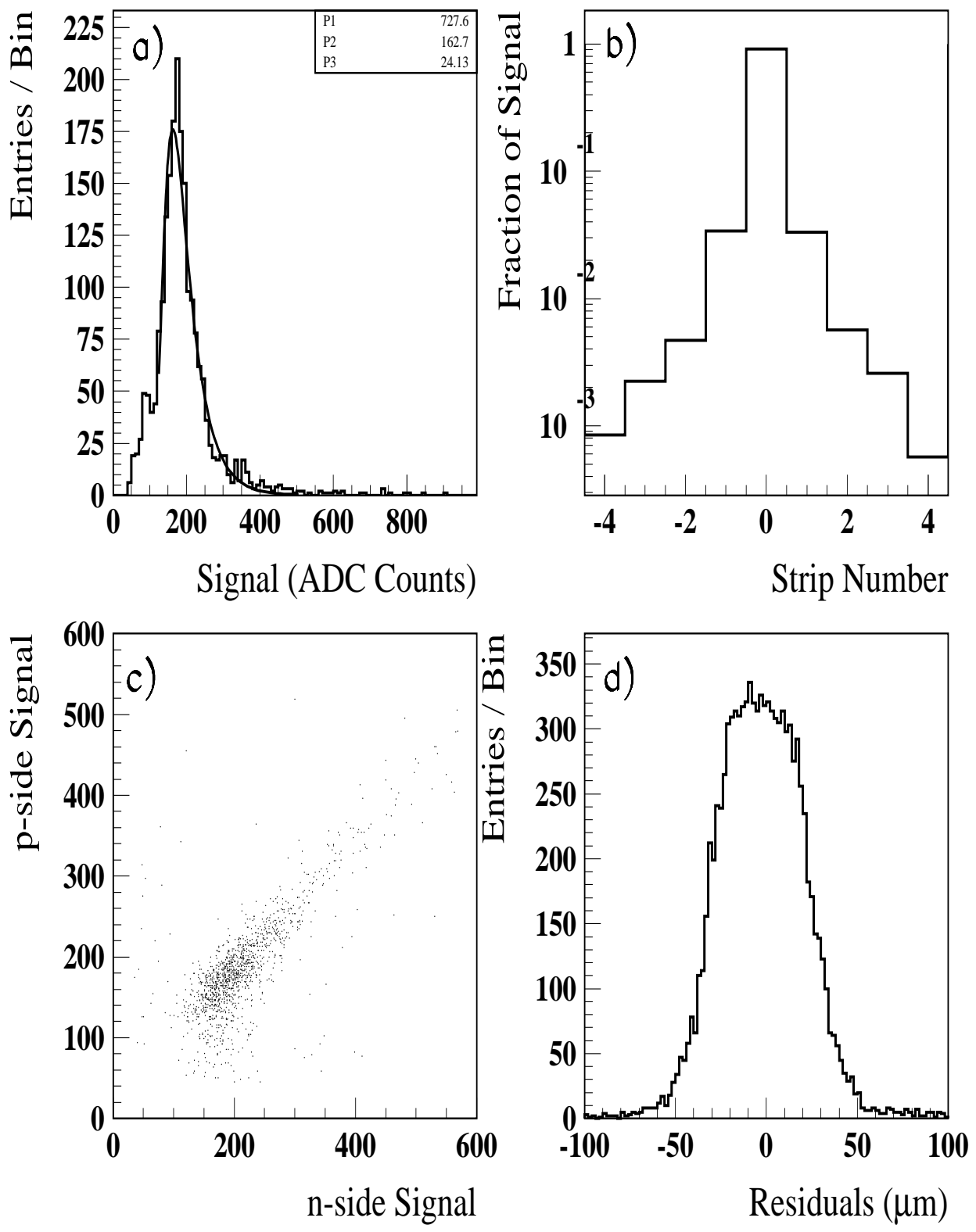

Figure 25: Data for DSODM: a) n-side Cluster Signal; b) n-side Cluster shape; c) p-side and n-side signals correlation; c) n-side Residual distribution. 

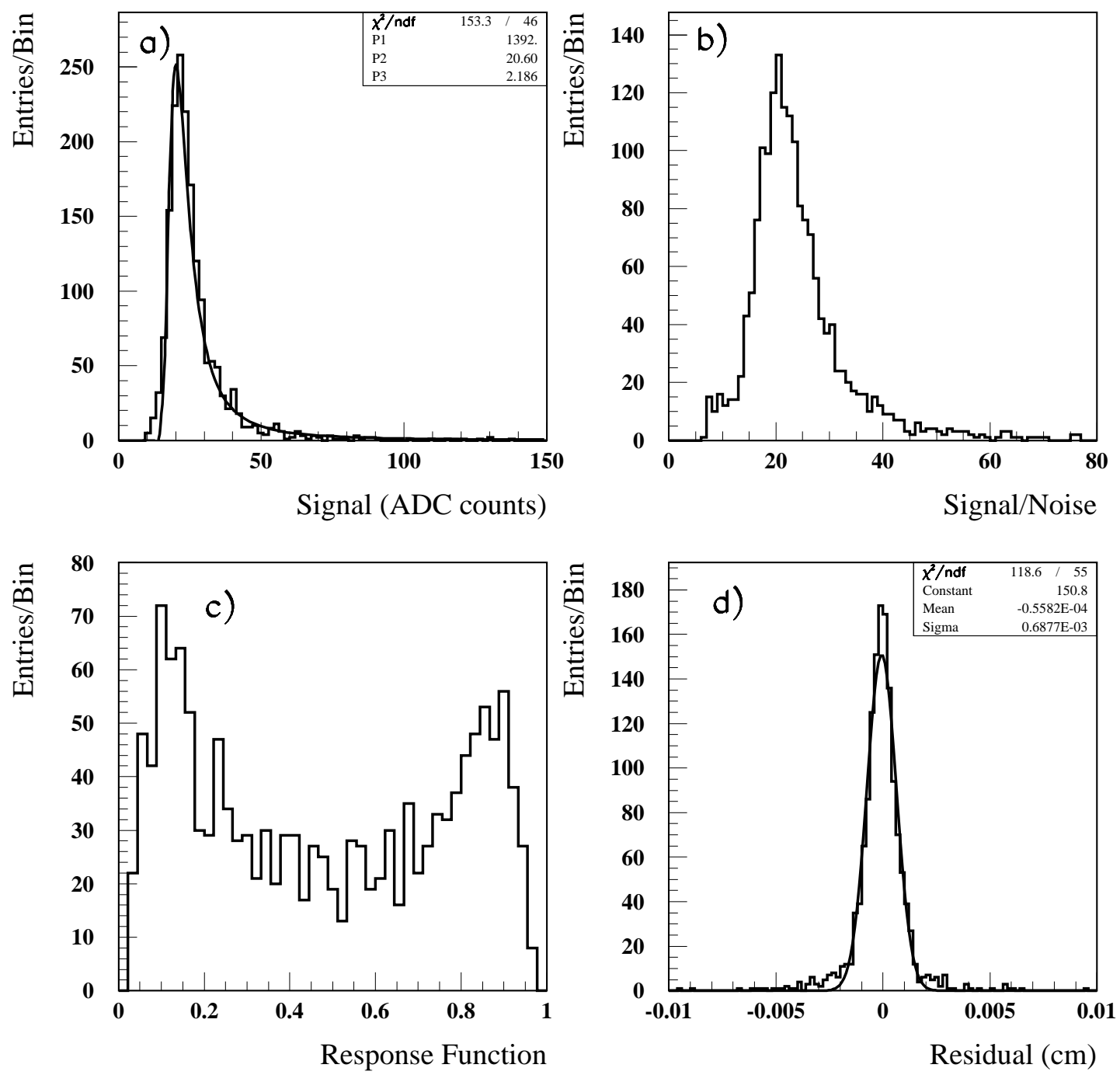

Figure 26: Distributions for the n-side of BABY: a) Cluster Signal; b) Signal to Noise ratio; c) Response Function; d) Distribution of Residuals. 

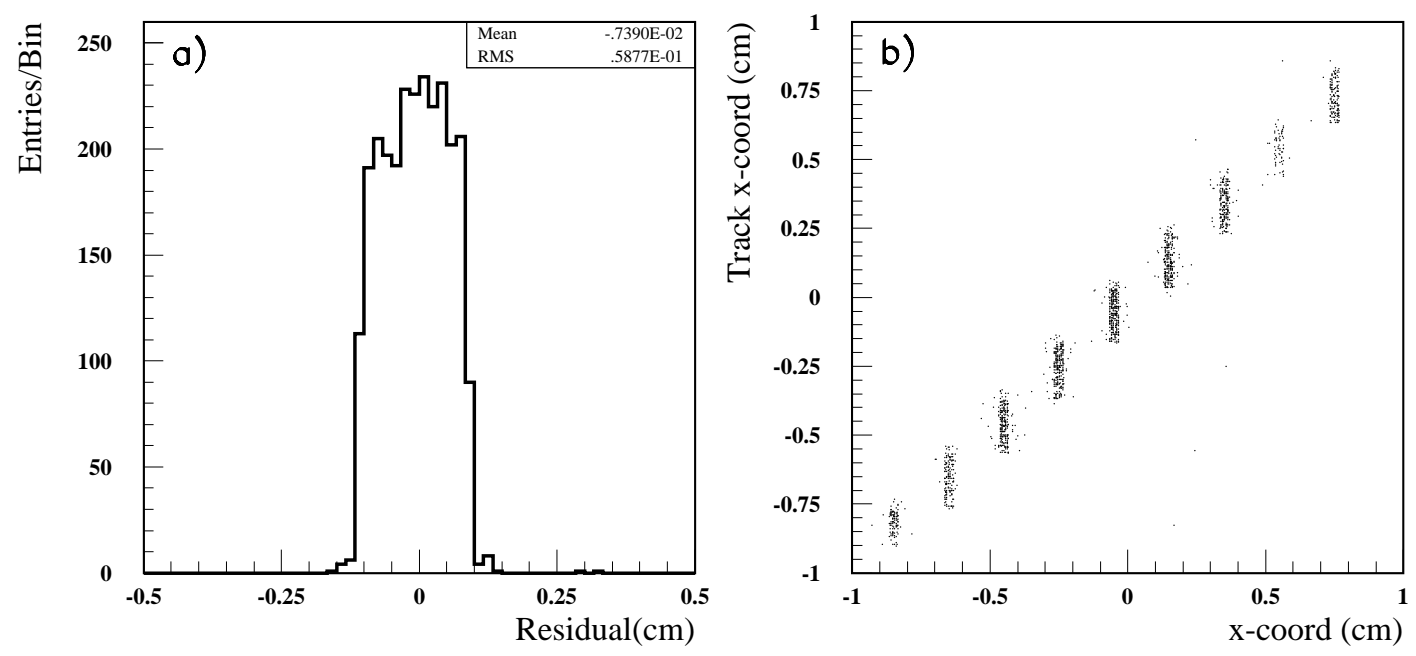

Figure 27: PAD: a) Residual distribution; b) Correlation with tracks reconstructed by the telescope. 

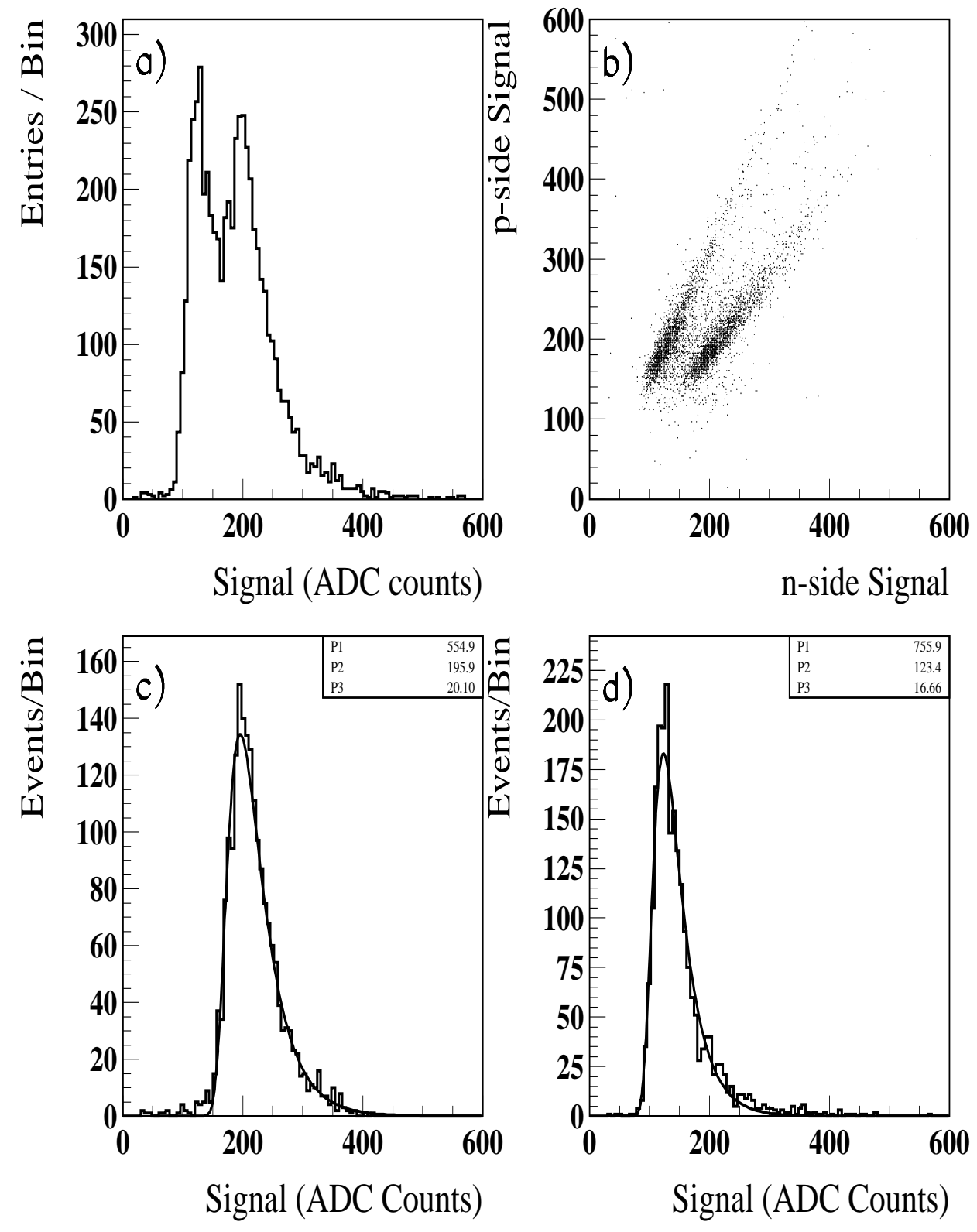

Figure 28: Distributions for the WEDGE n-side a) Cluster Signal; b) Cluster Signal correlation; c) Cluster Signal for single-strip clusters; d) Cluster Signal for multi-strip clusters. 

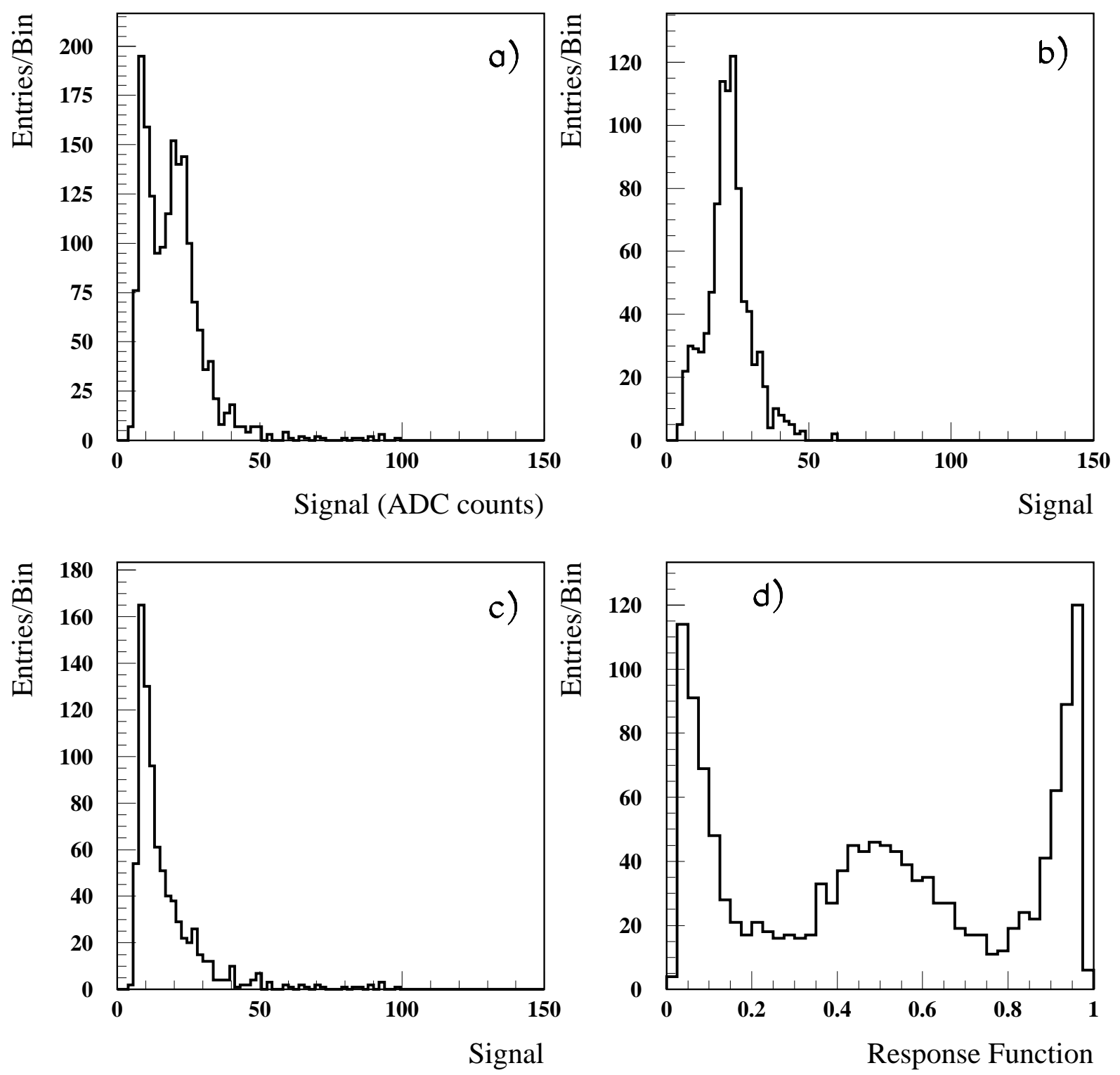

Figure 29: Distributions for the BABY p-side: a) Cluster Signal; b) Cluster Signal for single-strip clusters; c) Cluster Signal for multi-strip clusters; d) Response Function. 

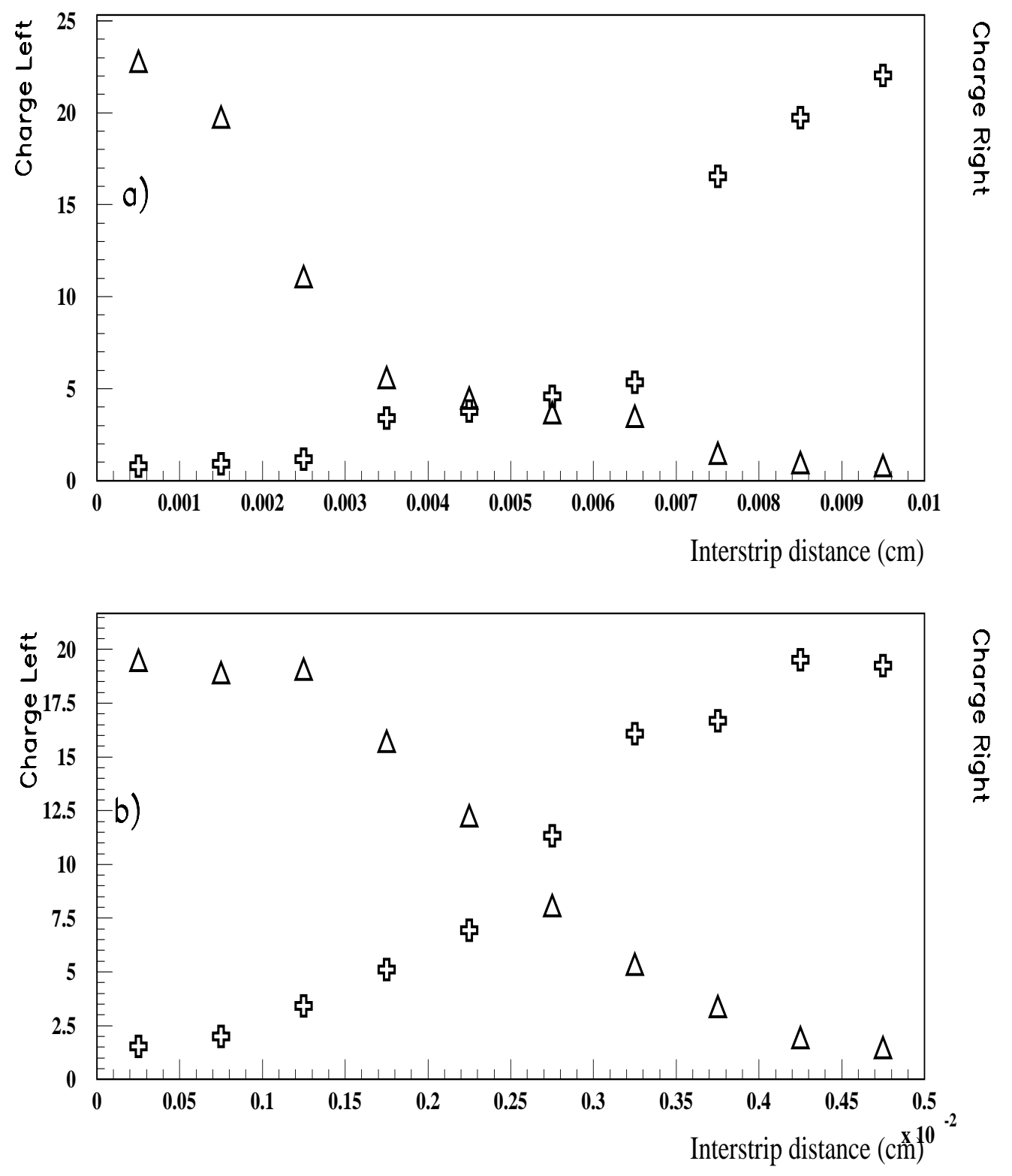

Figure 30: Data from BABY. Charge collected on the left and right strips (ADC counts) a) p-side ; b) n-side ; All variables are plotted versus the reconstructed position modulo the strip pitch. 


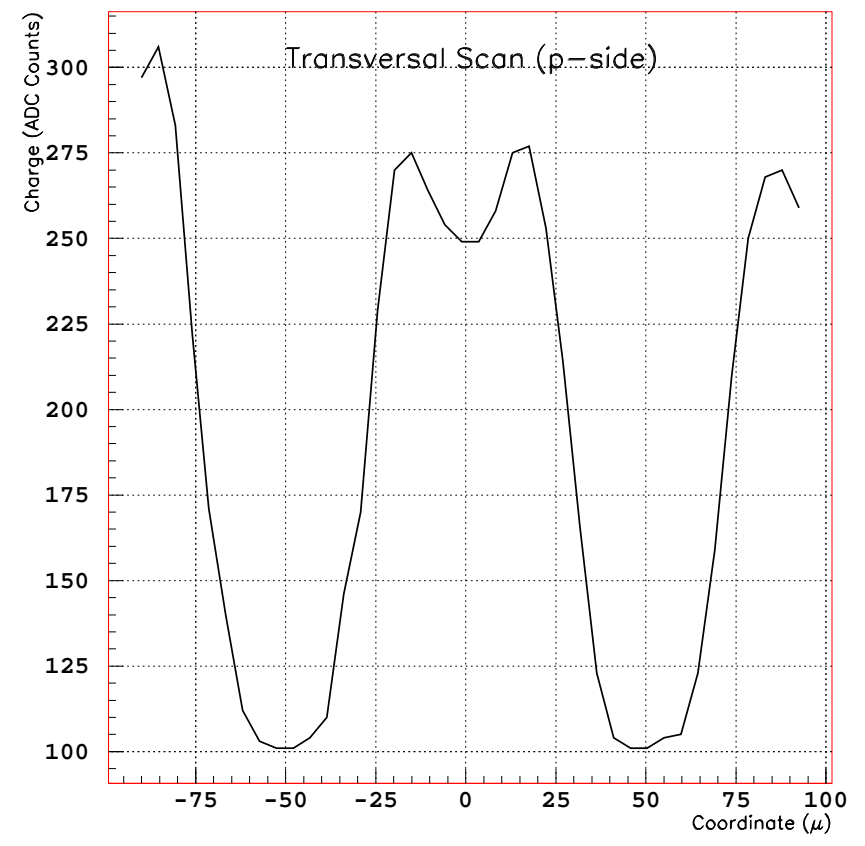

Figure 31: Cluster signal in the laser transverse scan; 